\title{
HYBRID ATOMISTIC-CONTINUUM SIMULATION OF NUCLEATE BOILING WITH DOMAIN RE-DECOMPOSITION METHOD
}

\author{
A Thesis presented to \\ the Faculty of the Graduate School \\ at the University of Missouri
}

In Partial Fulfillment

of the Requirements for the Degree

Master of Science

by

BO ZHANG

Dr.Chung-Lung Chen, Thesis Supervisor

JULY 2017 
The undersigned, appointed by the Dean of the Graduate School, have examined the dissertation entitled:

\section{HYBRID ATOMISTIC-CONTINUUM SIMULATION OF NUCLEATE BOILING WITH DOMAIN RE-DECOMPOSITION METHOD}

Presented by Bo Zhang, A candidate for the degree of Master of Science And hereby certify that, in their opinion, it is worthy of acceptance.

Professor Chung-Lung Chen

Professor Yuwen Zhang

Professor Stephen Montgomery-Smith 


\section{ACKNOWLEDGMENTS}

I would like to thank all the people who contributed to this research work. First and foremost, I would like to express my sincere gratitude to my advisor Prof. ChungLung Chen for the continuous support of my graduate study and related research, for his patience, motivation, and immense knowledge. His guidance helped me all the time in this research. He engaged me in new ideas, demanded a high quality of work towards the master degree, and supported me to publish a journal paper. Additionally, I would like to thank my committee members Professor Yuwen Zhang and Stephen Montgomery-Smith for their interest in my work.

The numerical model and results described in this thesis were accomplished with the help and support of the collaborators. Yijin Mao, a postdoctoral scholar worked together with me in this project, helped me to get familiar with OpenFOAM and LAMMPS. He always guides and encourages me to keep working. Without his efforts, my work would have undoubtedly been more difficult to accomplish. In addition, my sincere thanks also go to Hsiu-Hung Chen and Sheng Wang, who are also the postdoctoral scholars in my lab. They were working on the experimental study of this project. Without their experimental results, this project would be hard to be completed. And I would like to thank Zheng Li and Zhaoqing Ke, who helped me revise my paper and gave me insight advise. Besides these postdoctoral scholars, I would like to give my thanks to Haolun $\mathrm{Xu}$ and Kuojing Li, who are also the graduate students working in this lab. We encouraged each other on the graduate study including research and course work.

I am grateful for the funding source that allowed me pursue my graduate school study, Office of Naval Research (ONR). They funded this project and gave me the opportunity to develop my idea. I would like to acknowledge the Department of Me-

chanical and Aerospace Engineering at University of Missouri-Columbia. My gradu- 
ate experience benefitted greatly from the courses I took, such like Multiphase Heat Transfer, Computational Fluid Dynamics, the opportunity that I had served as a teaching assistant under Prof. Winholtz, and the high-quality seminars organized by the department. I would like to thank those working on University of Missouri Lewis3 high performance computing cluster, which a lot of my simulations ran on.

Finally, I would like to acknowledge my family and friends who supported me during my time here. I would like to thank my parents for their constant love and support, my wife, Qiwen Guo, who helped me with my research and courses, encouraged me to not give up, my friends, Run Yan and Chi Zhang, who made my time here at MIZZOU a lot of fun. 


\section{TABLE OF CONTENTS}

ACKNOWLEDGMENTS ................. ii

LIST OF TABLES $\ldots \ldots \ldots \ldots \ldots \ldots$ vi

LIST OF FIGURES $\ldots \ldots \ldots \ldots \ldots \ldots$ vii

NOMENCLATURE $\ldots \ldots \ldots \ldots \ldots \ldots \ldots$ ix

ABSTRACT ................... xiii

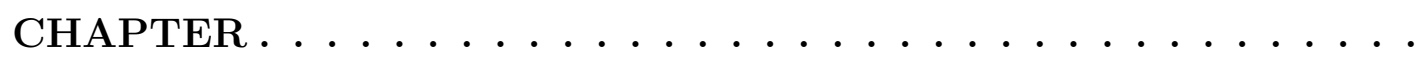

1 INTRODUCTION $\ldots \ldots \ldots \ldots \ldots \ldots \ldots \ldots$

1.1 Background and Literature Review . . . . . . . . . . . . . 1

1.2 Molecular Dynamics Simulation of Multiphase Problems . . . . . . 3

1.2 .1 Surface Tension $\ldots \ldots \ldots \ldots \ldots \ldots \ldots$

1.2 .2 Tolman Length . . . . . . . . . . . . . . . . . . 4

1.2 .3 Line Tension . . . . . . . . . . . . . . . 5

1.3 Conventional Hybrid Atomistic-continuum Method . . . . . . 7

1.4 Objective of the Present Work $\ldots \ldots \ldots \ldots$

2 METHODOLOGY .................... 11

2.1 Fixed decomposition hybrid simulation $\ldots \ldots \ldots \ldots$

2.1 .1 MD Simulation . . . . . . . . . . . . . . 12

$2.1 .2 \quad$ CFD Simulation. . . . . . . . . . . . . . . . . 13

2.1.3 CFD-MD Coupling Schemes _. . . . . . . . . . . 13

2.2 Hybrid atomistic-continuum method . . . . . . . . . . 20

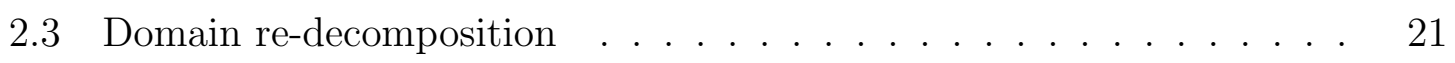

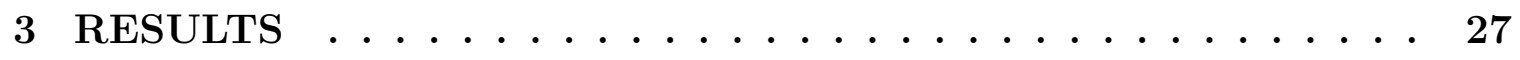


3.1 Model Validation . . . . . . . . . . . . . . . . . . . . 27

3.1.1 Sudden Start Couette Flow . . . . . . . . . . . . . . . . 27

3.1.2 Couette Flow Simulation with Domain Re-decomposition . 30

3.2 Nucleate Boiling on Structured Surface . . . . . . . . . . . . . . . 31

4 CONCLUSION AND DISCUSSION . . . . . . . . . . 42

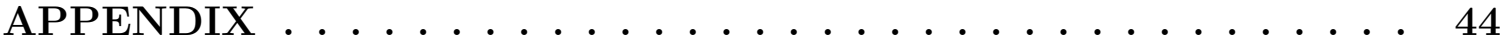

A Coupling Loop ...................... 44

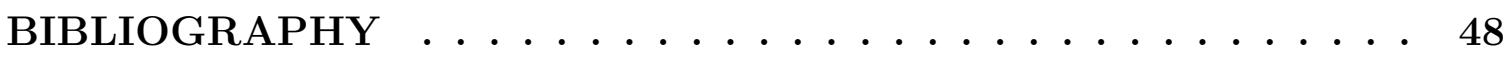




\section{LIST OF TABLES}

Table $\quad$ Page

3.1 Argon fluid properties at $116 \mathrm{~K}, 1 \mathrm{MPa}$. . . . . . . . . . . . 32 


\section{LIST OF FIGURES}

Figure $\quad$ Page

1.1 Principal stresses $P_{T}$ and $P_{N}$ near the liquid-vapor interface . . . . 4

1.2 Three-phase line in a three phases system . . . . . . . . . . . 6

1.33 -D droplet and 2-D droplet . . . . . . . . . . . . 7

1.4 Hybrid atomistic-continuum simulation domain . . . . . . . . . . . 9

1.5 Schematic of dynamic re-decomposition method for film boiling. (a) The initial CFD, MD region and HSI (b) domain is not changed (c) domain is re-decomposed . . . . . . . . . . . . . . . 10

2.1 Schematic of the HSI with control region. The control region is defined at the top of HSI . . . . . . . . . . . . . . . . . . 14

2.2 Equilibration of control region. (a)Initial control region (b) Atoms are inserted. (c) Atoms are equilibrated . . . . . . . . . . . . .

2.3 Variation of the average potential energy of argon atoms. The change of the atoms number occurs at the 100th time step. (a) Atoms are inserted. (b) Atoms are deleted ............... . . 24

2.4 Hybrid computational domain with a trigger region. . . . . . . . . 25

2.5 Domain re-decomposition at different conditions. (a) The bubble is too far from the HSI (b) The bubble is too close to the HSI. The dash line is the original MD domain. . . . . . . . . . . . . . . . 
3.1 Coupling simulation domain for Couette flow with heat transfer. On the right shows the geometry height, with the liquid-solid contact sur-

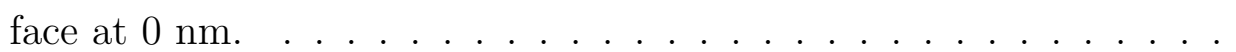

3.2 Comparison of pure CFD simulation and hybrid simulation results. Pure CFD results are shown in lines. The points are MD and CFD results in coupling method. (a) Velocity profile for simulation without re-decomposition (b) temperature profile for simulation without redecomposition (c) velocity profile for simulation with re-decomposition (d) temperature profile for simulation with re-decomposition . . . . .

3.3 Coupling simulation domains at different times. The re-decomposition

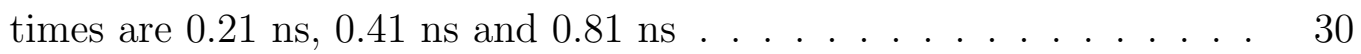

3.4 The geometry of the hybrid simulation case for nucleate boiling . . . 32

3.5 The potential energy of argon atoms during heating. (a) Potential energy right after equilibration (b) potential energy at $1.1 \mathrm{~ns} \quad .$. .

3.6 Nucleate boiling on structured surface at $130 \mathrm{~K}$. The CFD region shows the temperature distribution, and the MD region shows the atoms potential energy. . . . . . . . . . . . . . . . . . 34

3.7 Three-phase contact region . . . . . . . . . . . . . . . . 35

3.8 Nucleate boiling on structured surface at $140 \mathrm{~K}$. The CFD region shows the temperature distribution, and the MD region shows the atoms potential energy. . . . . . . . . . . . . . . . . .

3.9 Number of atoms variation over time. (a) Wall temperature is $130 \mathrm{~K}$ (b) Wall temperature is $140 \mathrm{~K} \ldots \ldots 37$

$3.10 \mathrm{CFD}$ and MD domains at different time steps . . . . . . . . . 38 
3.11 Comparison of atoms number in simulation. The dash line show the estimated atoms change if a fixed decomposition method is used for the same case. (a) Wall temperature is $130 \mathrm{~K}$ (b) Wall temperature is

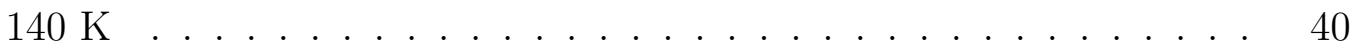

3.12 The cumulative energy flux flowing into the system . . . . . . . . 41

4.1 Domain decomposition when multiple bubbles exist . . . . . . . . 43 


\section{Nomenclature}

$A_{j} \quad$ cross-section area

$C_{p} \quad$ heat capacity

E Lennard-Jones potential energy

$f \quad$ conservative force on atom

F $\quad$ Gibbs free energy

I identity matrix

$k \quad$ Boltzmann constant

$L \quad$ the length of three-phase line

$m$ mass of atom

$\hat{n} \quad$ the unit normal pointing out of the surface

$N \quad$ total number of atoms

$p \quad$ pressure of the fluid

$P \quad$ principle stress in the fluid

$r \quad$ distance between two atoms 
$r_{c} \quad$ cutoff distance

$R$ radius of the bubble/droplet

$S \quad$ interface area

$T \quad$ coupling interval

$\bar{u} \quad$ average velocity of molecules

$u_{i f} \quad$ normal velocity at the boundary of CFD

$\bar{U}_{0} \quad$ atoms initial average potential energy

$\bar{U}_{t} \quad$ atoms average potential energy at time $t$

$v \quad$ atom velocity

$V \quad$ region volume

$Z \quad$ atoms number to be inserted

\section{Greek Symbols}

$\gamma \quad$ surface tension

$\delta \quad$ tolman length

$\epsilon \quad$ depth of potential well

$\theta \quad$ contact angle

$\mu \quad$ dynamic viscosity

$\rho \quad$ fluid density

$\sigma \quad$ zero-crossing distance for the potential

$\tau \quad$ line tension 


\section{Subscripts}

$i \quad$ index $i$

$j \quad \operatorname{index} j$

$l \quad$ liquid phase

$N \quad$ normal direction

$T \quad$ tangential direction 


\section{ABSTRACT}

The bubble nucleation plays a pivotal role in the boiling process. In order to have a comprehensive understanding of this phenomenon, a critical consideration on fluid-solid interaction at atomistic level is imperative. However, traditional Molecular Dynamics simulation requires prohibited amount of computational efforts to accomplish a full scale study. Hybrid atomistic-continuum method is a promising solution for this problem. It limits the atomistic region to a small scale where detailed information is preferable, while using continuum method for the rest of the domain. Nevertheless, none of the current hybrid method is suitable for solving a rapid expanding system like the bubble nucleation. In this study, a domain re-decomposition hybrid atomistic-continuum method is developed to conduct a multiscale/multiphase investigation on the bubble nucleation. In addition to the conventional coupling scheme, this method is capable to re-partition the molecular and continuum domain once it is necessary during the simulation. That is, the Computational Fluid Dynamics (CFD) and Molecular Dynamics (MD) regions are interchangeable on the fly such that the bubble is absolutely confined within MD region. Giving the fact that accurate modeling of interface tracking and phase change are still problematic for continuum mechanics on microscale, our coupling method directly avoids these issues since CFD domain takes care of a single-phase flow while the molecular domain simulates the bubble growth. With this idea in mind, this approach enables us to investigate the nucleate boiling on nanostructured surface with higher resolution than complete continuum mechanic model based simulation. In the present result, it is observed that bubble grows at a curved surface imposed with a constant super heat after nucleate boiling occurs. Meanwhile, the energy flux flows from solid to fluid is measured during the entire process. It is believed that this coupling method is very promising in studying nano-bubble related multiphase problems on microscale. 


\section{Chapter 1}

\section{INTRODUCTION}

\subsection{Background and Literature Review}

Liquid-vapor phase change phenomena are frequently encountered in many engineering applications. It allows a fast heat removal from high performance electronic device, which is widely utilized in almost the entire field of engineering and everyday lives. However, the physics behind bubble nucleation and subsequent evaporation process are not well understood. To some extent, it impedes the prominent trends of miniaturization of electronic devices, which is essential for NEMS/MEMS. Although a few models had been proposed/implemented to enable the simulation of boiling in CFD code and obtains reasonable results [1-3], almost none of them has considered the complex interactions between micro structures and the surrounding fluid from the atomistic level, which are critical for bubble nucleation on solid surface. Moreover, some of them even need user to prescribe empirical coefficients which have to be carefully tuned based on case-dependent experiments in order to produce a good result [1]. Another fact is that those models are usually developed for solving macroscopic problems; they might not be suitable for microscale simulation of 
phase change phenomena because of the emergence of strong non-equilibrium state and discontinuity in the process.

Molecular dynamics (MD) is a computational method for studying scientific and engineering problems by simulating the motion of all single atom in a computational domain. It is capable of providing an atomistic level insight into nanoscale where continuum assumption breaks down. Previous studies indicate that this approach has been used to unveil the molecular nature of many multiphase problems. Mecke et al. [4] simulated a liquid-vapor interface of Lennard-Jones fluid, and obtained the co-existing densities and surface tension. Alejandre et al. [5] used MD to measure the surface tension of water from $316 \mathrm{~K}$ to $573 \mathrm{~K}$, and the results agreed well with experiments. Nijmeijer et al. [6] also studied the fluid interface and it was proved that the truncated tail of the potential is very important for the evaluation of surface tension. Zhou et al. [7] simulated the impingement of droplet on a liquid film. Bourg et al. [8] studied the noble gas diffusion in ambient liquid water. Kalikmanov et al. [9] compared the Tolman length theory with simulation result.

In addition, some studies considered the solid-fluid interactions. Werder et al. [10] investigated the contact angles of water droplet on carbon nanotubes and non-wetting behavior was observed. Koishi et al. [11] simulated a large scale transition between Wenzel State and Cassie State on hydrophobic surface. They also investigated the contact angle hysteresis for droplets on nanopillared surface [12]. Vrancken et al. [13] demonstrated a simple design paradigm that allowed for reversible switching of wetting states with electrowetting approach. Noorian et al. [14] simulated the channel flow passing walls with nanoscale roughness. Sharma et al. [15] computed the evaporation rate of water in hydrophobic confinement. Chen et al. [16] predicted the solid-liquid interaction from the wetting phenomena. Weijs et al. [17] investigated the line tension when three phases coexisted. Carey et al. [18] and Hu et al. [19] measured the disjoining pressure in ultra-thin liquid films. Hu et al. [20] investigated 
nanofluids with nonequilibrium MD simulation. There were also a few attempts to study bubble generation. Maruyama et al. [21] simulated a heterogeneous bubble nucleation by expanding two surfaces that bounds the fluid. Nagayama et al. [22] simulated a spontaneous bubble nucleation by creating a system with metastable fluid as an initial configuration. These bubble-liquid systems were different from heat induced boiling, but the phase change process under their conditions was successfully reproduced. The numerous results from MD method indicate some basic physical phenomena can be well simulated when the fluids are represented by molecules.

\subsection{Molecular Dynamics Simulation of Multiphase Problems}

\subsubsection{Surface Tension}

When an interface exists between liquid and vapor phases, the difference between the capillary forces across the liquid-vapor interface will result in a surface tension. From thermodynamics definition, surface tension is the work done per unit area by free energy to create new surfaces. For both droplets and bubbles, the surface tension intends to minimize the surface free energy by decreasing its area [23]. Kirwood and Buff were the first to derive expressions to relate surface tension with intermolecular potential and distributed functions [24]. They calculated the principal stresses $P_{T}$ and $P_{N}$ near a liquid-vapor interface, where $P_{N}$ is the stress normal to the interface, and $P_{T}$ is the tangential stress, as shown in Figure 1.1. Then the formula for surface tension can be written as

$$
\gamma=\int_{r_{1}}^{r_{2}}\left[P_{N}(z)-P_{T}(z)\right] d z
$$

By doing this, it is assumed that the two points are sufficiently far from the 
interface. The idea behind this method is that the stress tensor is anisotropic in and only in the liquid-vapor transition region [25]. In the region far from the interface, both $P_{T}$ and $P_{N}$ are pressure-like. Therefore, the stress difference is all owning to the surface tension effect, and its value can be calculated by an integration.

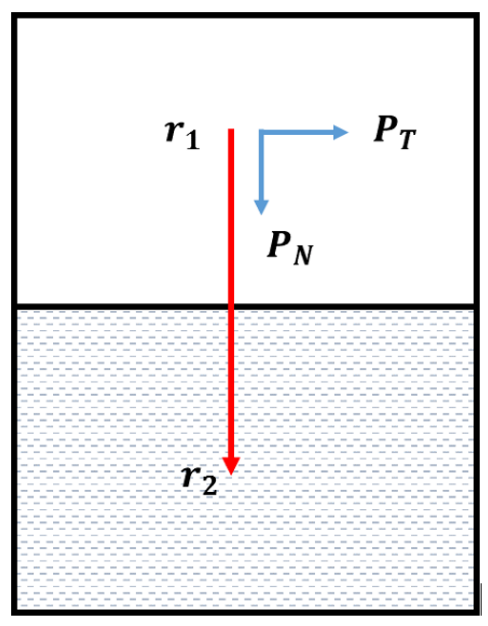

Figure 1.1: Principal stresses $P_{T}$ and $P_{N}$ near the liquid-vapor interface

\subsubsection{Tolman Length}

In macroscale multiphase problems, Young-Laplace equation is widely used to describe the pressure difference as a function of the surface shape.

$$
\Delta p=-\gamma \nabla \cdot \hat{n}=\gamma\left(\frac{1}{R_{1}}+\frac{1}{R_{2}}\right)
$$

Where $\Delta p=p_{\text {in }}-p_{\text {out }}, \gamma$ is the surface tension, and $R_{1}$ and $R_{2}$ are the principle radiuses of the surface. When modeling small droplets or bubbles, the size effect on surface tension has to be taken into account. In many simulations, $\gamma$ is defined as a constant, as long as the temperature and pressure of the system is not significantly changed. However, this assumption breaks down as the bubble size becomes very 
small. For a sphere bubble, the pressure difference equation has to be modified as

$$
\Delta p=\frac{2 \gamma}{R}\left(1-\frac{\delta}{R}+O\left(R^{-2}\right)\right)
$$

$\delta$ is the Tolman Length, named after Tolman in 1949 [26]. Tolman Length is defined as the distance between the location of the surface of tension and of the equimolar dividing surface. The modification can also be interpreted as the surface tension varies as a function of the radius.

$$
\gamma(R)=\gamma\left(1-\frac{2 \delta}{R}+O\left(R^{-2}\right)\right)
$$

Song et al. [27] performed molecular dynamics simulation for small liquid drops of argon. The pressure tensors and density profiles were monitored in the process. From the results, the radius of equimolar surface and the radius of surface of tension are obtained. Then the Tolman Length can be directly calculated. Though molecular dynamics can be exploited to provide foundations for other modeling approaches, the method itself can best represent the real physics related to this process.

\subsubsection{Line Tension}

In the interfaces between different material phases, there is a special region where all three phases contact with each other. This region is referred to as the three-phases line, as shown in Figure 1.2. The structure incurs interfacial forces that distort the interface, and consequently change the bubble or droplet dynamics [28]. Therefore, the theory of surface tension is no longer sufficient for this condition.

In order to describe this effect, the concept of line tension was introduced by Gibbs and it is analogized with surface tension [29]. Line tension is defined as the work done per unit length by free energy to create new contact line. If the bulk fluid energy, 


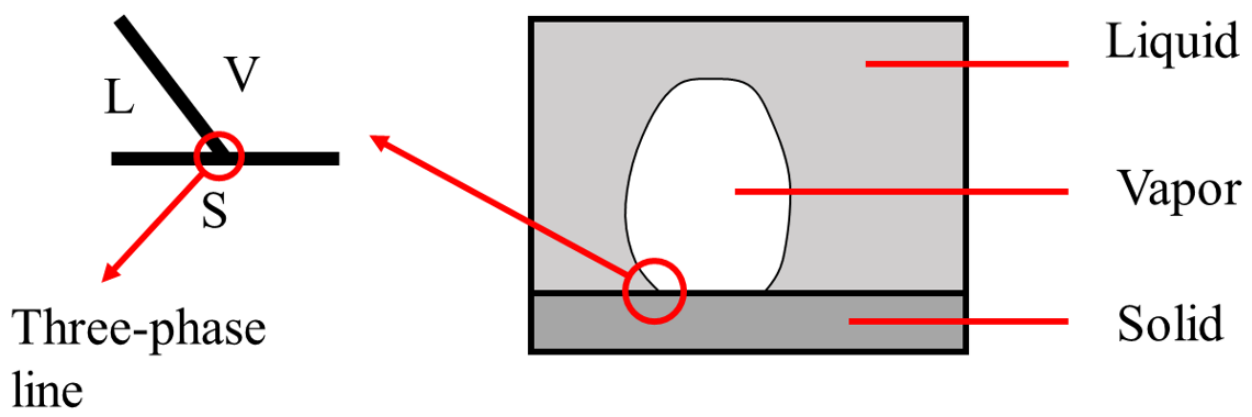

Figure 1.2: Three-phase line in a three phases system

surface tension and line tension are all considered at the same time, the system free energy can be written as

$$
d F=p d V+\sum_{i} \gamma_{i} d S_{i}+\tau d L
$$

Where $S_{i}$ is the interface area, $\gamma_{i}$ is the surface tension, and $\tau$ is the line tension, and $L$ is the length of three-phase line. Weijs et al. [17] described a method to measure line tension with molecular dynamics. Assuming there are two configurations for liquid on solid surface, as shown in Figure 1.3. The spherical caps cross section areas are the same for the two cases. From equation 1.5, the contact angle for the first case is stable when the free energy is minimized.

$$
\cos \theta_{1}=\frac{\gamma_{S V}-\gamma_{S L}}{\gamma}-\frac{\tau}{\gamma R}
$$

$R$ is the radius of the liquid-solid contact circle. The droplet size remains the same all the time. This equation takes both surface tension and line tension into account. For the second case, the three-case line is a straight line. Its length cannot be changed by an energy minimization. So the contact angle is given by only reflecting the surface tension.

$$
\cos \theta_{2}=\frac{\gamma_{S V}-\gamma_{S L}}{\gamma}
$$


The line tension can be calculated by comparing equations 1.6 and 1.7 .

$$
\tau=\gamma R\left(\cos \theta_{2}-\cos \theta_{1}\right)
$$
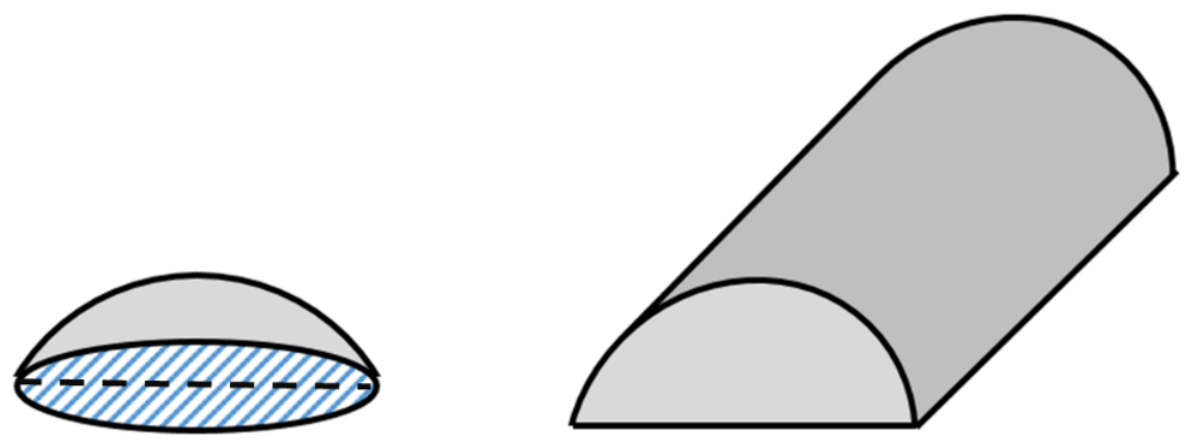

Figure 1.3: 3-D droplet and 2-D droplet

\subsection{Conventional Hybrid Atomistic-continuum Method}

Despite the continuous progress of molecular dynamics simulation in the past decades, a further study of bubble growth is still impeded by its extremely high demand for computational resource. Currently, most of molecular domains are limited to tens of nanometers, within which a stable bubble can barely be observed. As a result, those studies usually focus on a specific region of a multiphase system. However, if computational cost is not a problem, a full MD simulation of bubble growth is more desirable. Another difficulty for MD simulation is associated with the boundary condition. Phase change leads to rapid volume expansion or shrink, therefore an open boundary that allows inflow and outflow of molecules is necessary. It is worth mentioning that inflow/outflow for finite volume method is quite natural while it is difficult for molecular dynamics simulation [30]. Due to these facts, a concept of hybrid atomistic-continuum methodology has been proposed to take the advantage of both methods [31]. This methodology combined CM (continuum mechanics) and MD 
approaches, and potentially it is capable to solve challenging problems on microscale, which is out of reach for full molecular dynamics simulation. Figure 1.4 illustrates a typical geometry for a hybrid atomistic-continuum simulation. As shown, the entire fluid domain is decomposed into continuum and particle regions while the hybrid simulation interface (HSI) is used to exchange data between these two regions. A literature review shows that hybrid atomistic-continuum method is mostly used to study near wall effect on fluid flow on micro/nano-scale [31-34]. Since MD approach is more time consuming, only the flows near solid walls are modeled by particles to minimize the computational cost. In the first attempt, the hybrid method used available multiphase CFD models. The bubble nucleates from MD and grows. When it is big enough, the bubble interface penetrates the HSI, and CFD takes charge of the interface transport with Volume of Fluid (VOF) method. This is not easy to accomplish, though. MD method simulate the interface from a practical physical view, it shows the liquid-vapor transition region has a depth of several nanometers. However, in CFD, VOF method has a transition region with a depth of several cell lengths. The cell size may vary based on the problem size and applications.

If the interface depths are not equal in the two methods, the bubble penetration process cannot match well. In addition, once the bubble interface appears in CFD, all the previous concerns about the various CFD models come back, and the simulation will be less convincing in this way. If the process of bubble penetrating the HSI is avoided, many problems will no longer exist. In this case, the liquid-vapor interface also needs to be simulated by MD due to its complexity and discontinuity. The problem arises as the liquid-vapor interface is not fixed, and its shape and position vary as the bubble grows. If the interface is to be confined in the MD region, the HSI has to be placed sufficiently far apart from the solid surface. With this restriction, the simulation is only possible when the bubble is sufficiently small to stay within the scope of MD region; the only benefit of this coupling scheme is to provide an open 
boundary.

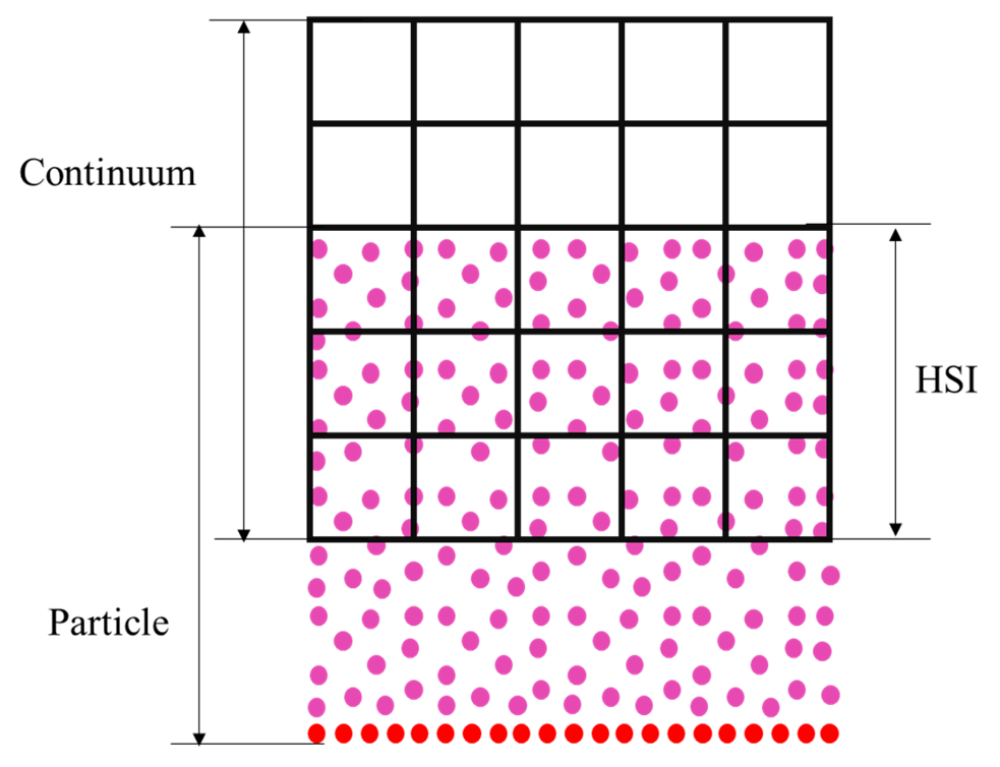

Figure 1.4: Hybrid atomistic-continuum simulation domain

\subsection{Objective of the Present Work}

This study explores to develop a multi-scale model depicting the liquid-vapor phase change phenomena with an expectation to enhance the capability of the present simulation tool. For overcoming the shortage in coupling method, a dynamic redecomposition method is introduced in this code. The objective is to ensure the interface stays in MD region, while minimizing the number of particles in the system. The method is illustrated in Figure 1.5. Initially, only the regions near the solid surface are simulated by MD. However, when the phase change occurs, the interface moves away from the wall and approaches HSI. At that moment, the domain is re-decomposed in order to use a larger MD region. The position of HSI is also changed according to the new boundaries. In a bubble growing simulation, this redecomposition method can be applied multiple times to accommodate the interface wherever/whenever necessary. 


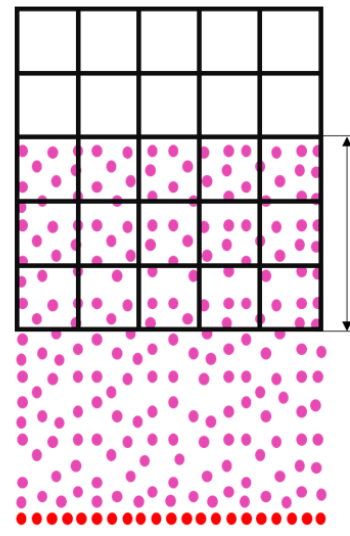

(a)

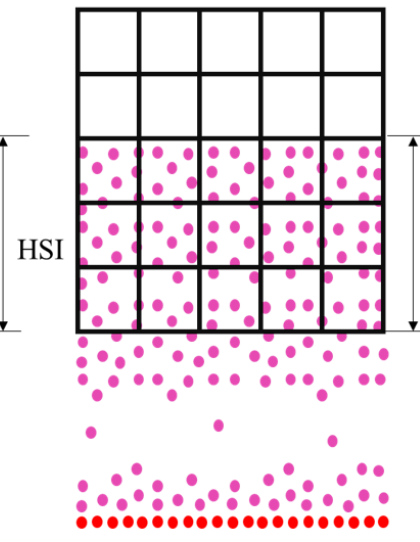

(b)

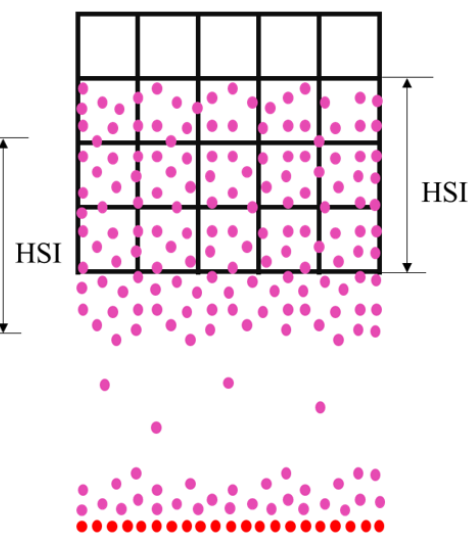

(c)

Figure 1.5: Schematic of dynamic re-decomposition method for film boiling. (a) The initial CFD, MD region and HSI (b) domain is not changed (c) domain is redecomposed 


\section{Chapter 2}

\section{METHODOLOGY}

The domain re-decomposition method is based on a conventional hybrid atomisticcontinuum approach, in which the subdomains, namely CFD and MD regions, are unchangeable during the computation. The simulation in the present work innovatively uses an adjustable hybrid simulation interface. In specific, the simulation follows the same way as the conventional fixed-decomposition method until the interface approaches the HSI and finally falls into a trigger zone to activate a re-decomposition process. After new HSI is determined, all the information from the original domain will be mapped into the new regions, then the simulation is resumed and carried on like a conventional hybrid method. In the following section, the fixed decomposition hybrid simulation will be introduced and then the principles for re-decomposition follows. 


\subsection{Fixed decomposition hybrid simulation}

\subsubsection{Simulation}

Molecular dynamics is a computer simulation method for the behavior of an atomic system. The simulation is performed by numerically solving Newtons equations of motion. For a simple system, it can be written as

$$
\begin{aligned}
& m_{j} \dot{v}_{j}=f_{j} \\
& f_{j}=-\frac{\partial E}{\partial r}
\end{aligned}
$$

$E$ is the atom potential energy. In this study, all particle interactions are dictated by the Lennard-Jones (LJ) potential.

$$
E=4 \epsilon\left[\left(\frac{\sigma}{r}\right)^{12}-\left(\frac{\sigma}{r}\right)^{6}\right] \quad r<r_{c}
$$

where $\epsilon$ is the depth of potential well, $\sigma$ is the distance at which the potential energy equals zero, and $r<r_{c}$ is the cutoff distance for particle interactions. In the following simulation, two types of atoms, argon and copper, are used to create fluid and metallic solid surface. For copper-copper interaction, the parameters are given as $\sigma=2.277$ $\AA$ and $\epsilon=0.415 \mathrm{eV}$, the potential parameters were determined from the known experimental values of cohesive energy and lattice constant at room temperature [35]. The argon-argon interaction is defined by Rahman's simulation [36], with $\sigma=3.405$ $\AA$ and $\epsilon=0.0103 \mathrm{eV}$. For the argon-copper interaction, the parameters are obtained in terms of the sixth-power mixing rule as

$$
\epsilon_{A r-C u}=\frac{2 \sqrt{\epsilon_{A r} \epsilon_{C u}} \epsilon_{A r}^{3} \epsilon_{C u}^{3}}{\epsilon_{A r}^{6}+\epsilon_{C u}^{6}}
$$




$$
\sigma_{A r-C u}=\left(\frac{\sigma_{A r}^{6}+\sigma_{C u}^{6}}{2}\right)^{1 / 6}
$$

All simulations are performed under NVE ensemble, the pairwise cutoff is $13 \stackrel{\circ}{A}$, and the time step is $5 f s$. The molecular dynamics is performed with LAMMPS opensource software package [37].

\subsubsection{CFD Simulation}

In order to facilitate the development of coupling schemes, the widely used CFD open-source software OpenFOAM [38] is employed. For this study the solver is only required to solve simple single phase laminar flow. The CFD domain is governed by incompressible Navier-Stokes equations. Since the vapor phase is intentionally

isolated from CFD region, no multiphase model is necessary. The momentum and energy equations employed are expressed as

$$
\begin{aligned}
\frac{\partial \vec{U}}{\partial t}+\nabla \cdot(\vec{U} \times \vec{U})=- & \frac{\nabla p}{\rho}+\nabla \cdot\left(\frac{\mu}{\rho} \nabla \vec{U}\right)+\left(\frac{\mu}{\rho}(\nabla \vec{U})^{T}-\frac{2}{3} \frac{\mu}{\rho} \operatorname{trace}(\nabla \vec{U})^{T} \mathbf{I}\right) \\
\frac{\partial T}{\partial t}+\nabla \cdot(\vec{U} T) & =\nabla \cdot\left(\frac{k}{\rho C_{p}} \nabla T\right)
\end{aligned}
$$

The pressure velocity coupling is solved with PISO (Pressure Implicit with Splitting of Operator) algorithm. The influences of heat transfer on fluid properties are negligible since the CFD region is placed far from the wall, and bubble is considered to nucleate only at surface where liquid and solid are in contact. Therefore, it is assumed that all fluid properties in CFD region remain constants.

\subsubsection{CFD-MD Coupling Schemes}

The most important component of hybrid atomistic-continuum method is the HSI where ideally mass, momentum, and energy should match seamlessly. For the cur- 
rent problem of interest, it is expected that mass flow across the CFD and MD would be significant due to the emerging of new bubbles. In order to simulate the mass flux across the HSI, the number of molecules within this region has to be adjusted. However, it is known that molecule insertion and removal will inevitably introduce noise into the molecular system. The spurious energy change can be overwhelming without special caution. Previous studies showed this effect could be reduced by applying a control region right above the HSI [39], as shown in Figure 2.1. The region is specially used for deleting or inserting molecules as needed. Adjacent to that, there are continuum to particle region, buffer region and particle to continuum region, which are responsible for matching the thermodynamics states in the overlap region. This approach separates the most fluctuating region from the main computational domain, and allows for sufficient controlling over the boundary conditions. The controlling schemes will be discussed in detail in the following. As the conventional coupling scheme, Schwarz iteration [40-42] is adopted to carry out the simulation by alternatively applying boundary conditions to each domain.

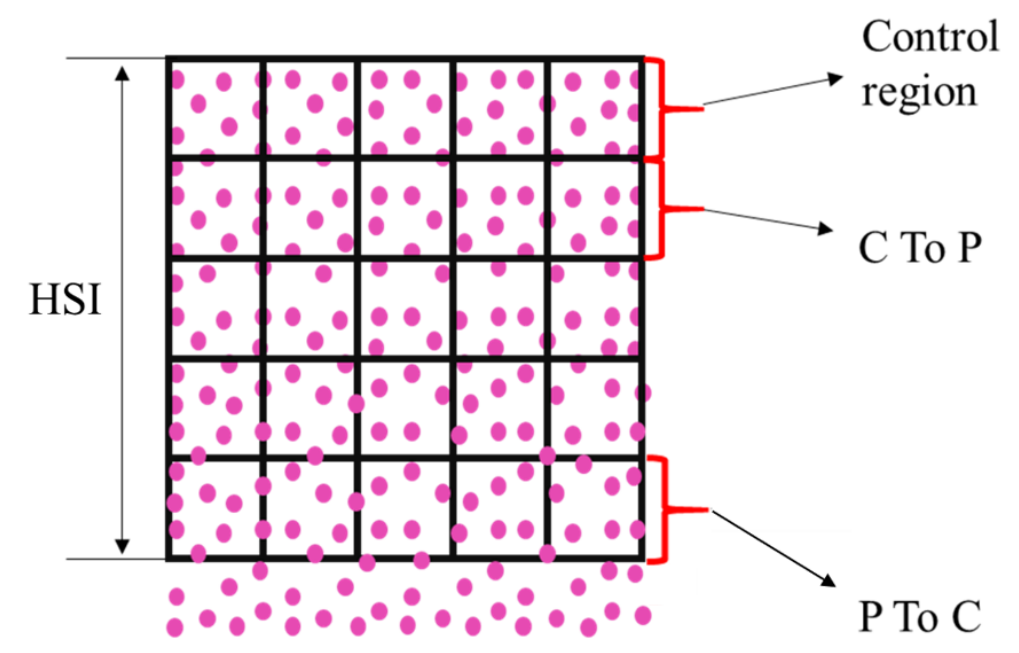

Figure 2.1: Schematic of the HSI with control region. The control region is defined at the top of HSI 


\section{Particle to Continuum (P2C) Schemes}

The Particle to Continuum (P2C) region is located at the CFD boundary in overlap region. It functions by reconstructing atomic information from MD domain to provide boundary conditions for CFD domain. The information to be transferred for single-phase incompressible flow includes velocity and temperature. For this purpose, the molecules in $\mathrm{P} 2 \mathrm{C}$ region are selected for the calculation of local velocity and temperature. For each element at the boundary, a square box centered at the boundary is defined. The velocity can be found through temporally averaging over all atoms within the box as

$$
\bar{u}=\left\langle\sum_{1}^{N} m_{j} v_{j} / \sum_{1}^{N} m_{j}\right\rangle
$$

The chevrons are used to denote an average over time, $m_{j}$ and $v_{j}$ are the mass and velocity of a single atom, and $N$ is the number of atoms in that region. To minimize the fluctuations in MD approach, each selected region should contain sufficient amount of atoms to produce a stable bulk velocity. The temperature in the MD region can be found through the kinetic theory as

$$
T=\left\langle\frac{2}{3 N k} \sum_{1}^{N} \frac{1}{2} m_{j}\left(v_{j}-\bar{u}\right)^{2}\right\rangle
$$

where $\bar{u}$ is the bulk velocity calculated in equation $2.8, k$ is the Boltzmann constant. Again, the value is averaged over the period of MD simulation.

\section{Continuum to Particle (C2P) schemes}

The Continuum to Particle (C2P) Schemes are account for the boundary of MD simulation. In comparison with $\mathrm{P} 2 \mathrm{C}$ scheme, it is more difficult to build a $\mathrm{C} 2 \mathrm{P}$ principle because of the dramatic inconsistency of degrees of freedom $(D O F)$ between

continuum and discrete descriptions. For a mono atomic system with $N$ particles, 
the reconstruction of microscope state requires $D O F_{M}$ of $6 N$ from macroscopic state where $D O F_{C}$ equals to $5\left(D O F_{M}\right.$ and $D O F_{C}$ are degrees of freedom for particle and continuum domains) [43]. Therefore, it is impossible to specify every degree of freedom for particles from the known states provided by CFD. As a compromise, external operations are introduced to match the macroscopic states, such as bulk velocity and temperature, with the ones from continuum domain. In the other words, at the end of each coupling interval, the sample atoms in $\mathrm{C} 2 \mathrm{P}$ region are expected to show the same average velocity and temperature as dictated in CFD domain. This can be achieved with constraint dynamics [31]. Specifically, in order to match the average velocity of atoms to CFD velocity, an additional force that is proportional to velocity difference will be applied to each atom within the constrained region,

$$
\dot{v}_{j}=k_{v}\left(u_{i}-\bar{v}_{i}\right)+\frac{f_{j}}{m_{j}}
$$

where $\bar{v}_{i}$ is the average atomic velocity, $u_{i}$ is the target velocity, and $k_{v}$ is the coefficient determining the strength of the constraint, $m_{j}$ is the mass of a single atom, $f_{j}$ is the conservative force associated with atomic interactions.

In the aspect of temperature, the velocity of each atom is modified by directly rescaling so as to meet the kinetic theory based temperature,

$$
v_{j}^{*}=\bar{v}_{i}+\sqrt{\frac{T_{C}}{T_{P}}}\left(v_{j}-\bar{v}_{i}\right)
$$

where $T_{P}$ is the kinetic temperature given by equation 2.9 , and $T_{C}$ is the target temperature from continuum domain. $v_{j}$ is the velocity of the atom, and $v_{j}^{*}$ is its velocity after rescaling. This scheme directly changes the atom velocity so that the temperature in this region equals to the target within a given coupling interval.

On the side of mass conservation, the control region is placed right above the $\mathrm{C} 2 \mathrm{P}$ region. Particles are inserted or removed to simulate the inflow or outflow at the MD 
boundary. And this operation depends on the mass flow rate at the same position of CFD domain. Since all the particles used here are monoatomic molecules, the number of atoms to be deleted or created within this control region is determined at the beginning of each coupling interval through

$$
Z_{i}=\frac{u_{i f} \rho_{l} A_{j} T}{m_{j}}
$$

where $u_{i f}$ is the normal velocity at MD boundary, which is extracted from CFD part of the overlapping region. $\rho_{l}$ is the liquid density, $A_{j}$ is the cross-section area, and $T$ is the equivalent time period of the coupling interval. The value $Z_{i}$ is rounded to its nearest integer to represent the variation of atoms, and the error caused by rounding is counted into the next coupling interval as an additional mass flux across HSI. Assuming a positive $u_{i f}$ points to the direction leaving MD domain, a positive $Z_{i}$ denotes the number of atoms to be deleted, and a negative $Z_{i}$ denotes atoms to be inserted.

Due to the lack of information from CFD domain, the exact positions of inserted and removed atoms are not specified. The most significant problem is the spurious change of potential energy. If atoms are inserted randomly, they may easily ruin the system and soon cause a complete failure of a simulation. If atoms are deleted randomly, small cavities, which disturb the energy distribution, are created around the spots where atoms are removed. To minimize the disturbance due to atoms number adjustment, the control region is equilibrated on the fly before a follow-up MD simulation. It should be noted that the atoms potential energy is not minimized, the goal of this step is to relax the atoms in given region to approach a normal state. The equilibration is a common method to prepare a molecular system before simulation. As well known, in most MD simulations, the initial arrangement of atoms is not appropriate since it is most likely not in equilibrium state. There are some high 
potential energy spots where atoms are too close to each other, and the uneven energy distribution causes abnormal behaviours. For example, the temperature may automatically increase as simulation goes on, since the high potential energy is gradually released when the fluid atoms are back to equilibrium. In addition, the equilibrate state of material is also a fundamental assumption in continuum approach, and thus being a pre-request for matching at overlap region. To find a better configuration, sufficiently long relaxation time to the atoms in the control region is required before performing any further operation. This procedure is currently employed as part of the coupling approach and is carried out after adjustment for number of atoms. As shown in Figure 2.2, at the beginning of atoms change, the whole MD domain is frozen (Figure 2.2(a)). The designated number of atoms change is applied to the control region, as shown in Figure 2.2(b). After that, the atoms in control region are released, and their bulk velocity is adjusted to zero. Then the control region is equilibrated with constraint dynamics (equation 2.11). This process gets rid of the abnormal energy within the system, while maintaining the temperature corresponding to CFD simulation because of the constraints (Figure 2.2(c)). Since all the atoms except those in control region are held in their original position, there is no coupling process happening in the main MD domain. The equilibration is not counted into the simulation time of fluid and heat transfer. In the end, the bulk velocity of atoms in each cell of control region is assigned with the corresponding values in CFD and all the constraints are removed before the follow-up MD simulation. After performing these steps, the new inserted atoms, surrounding atoms and those near the deleted atoms are no longer distinguishable from other existing ones. Therefore, it is expected that the influence caused by changing particle number can be reduced to some extent.

In the following, the potential energy disturbance due to the number control of atoms is examined over a $2 \mathrm{~nm} \times 2 \mathrm{~nm} \times 2 \mathrm{~nm}$ simulation box with periodic boundary 


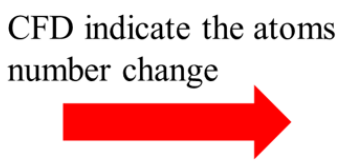

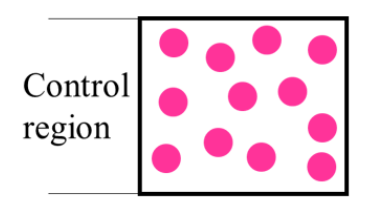

(a)

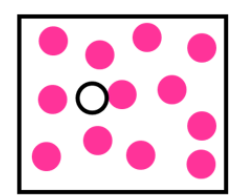

(b)

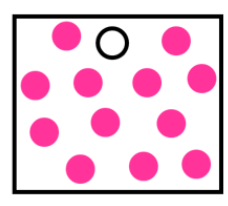

(c)

Figure 2.2: Equilibration of control region. (a)Initial control region (b) Atoms are inserted. (c) Atoms are equilibrated

conditions. Initially the system contains 150 argon atoms equilibrated at $110 \mathrm{~K}$, corresponding to a density of $1244 \mathrm{~kg} / \mathrm{m}^{3}$. The small case is studied since the cells in control region are usually much smaller than the whole MD domain in hybrid method. In order to simulate a mass flux entering MD domain, $1-10$ atoms $(0.67 \%-6.7 \%$ of the total atoms) are randomly inserted into the system. The insertion scheme forces a minimum distance of $0.22 \mathrm{~nm}$ between new and existing atoms. This constraint is applied in order to accelerate the energy adjustment. The point of insertion is randomly selected and tested until the coordinate meets the requirement. It takes only a few attempts since the required distance is much shorter than the average distance between atoms. Despite the limitation, the inserted atoms still have much higher potential energy compared with existing atoms. The problem is remitted by the energy minimization procedure which moves the new atoms along their force direction to a lower energy state. After that, all atoms are equilibrated for 200 time steps with constraint dynamics. Figure 2.3(a) shows the variation of the average potential energy of fluid atoms after insertion. The variation of potential energy is calculated by $\left(\bar{U}_{t}-\bar{U}_{0}\right) / \bar{U}_{0}$, where $\bar{U}_{0}$ is the initial average potential energy, and $\bar{U}_{t}$ is that for time $t$. The number of atoms is adjusted at the 100th time step and causes an enormous change of the potential energy. The horizontal lines give reference percentage changes of $5 \%, 0 \%$, and $-5 \%$. Since the original potential energy is negative, the increase of energy shows a negative percentage change. After a few time steps, the average potential energy tends to be stable. However, due to the 
density change, the mean potential energy does not converge to the original value and it deviates even more when more atoms are inserted. Upon inserting 10 atoms, the mean potential energy produces a deviation of $5 \%$ from the original one. Figure 2.3(b) shows the energy variation after atoms are removed. Though the deletion will not lead to a failure of simulation as atoms insertion, it still causes significant energy change. The influence can also be reduced by the process of equilibration. In this case, the energy change is about $5 \%$ after equilibration if 7 atoms (4.7\%) are removed from the system. Provided that only a small portion of the particles (less than $4.7 \%$ in this case) is modified each time, the method is generally helpful to stabilize the MD simulation where number of atoms changes.

\subsection{Hybrid atomistic-continuum method}

In a hybrid atomistic-continuum method, the simulation domain is decomposed into CFD and MD regions. And the HSI is defined where the two regions overlap. The critical part of this simulation is the bubble nucleation on the solid-liquid contact surface, where phase change happens as a result of continuous heating. Apparently MD should account for this region. In most hybrid methods, the particle region is very small, the bubble will touch the HSI soon after it begins to grow. The classical hybrid method has two difficulties in dealing with this situation. The first problem is related to the intrinsic flaws in CFD fluid models. For example, at nano-micro scale, the force caused by surface tension is considered to be a function of curvature, and it is also likely to depends on many other conditions. In addition, the existing liquid-vapor phase change models can hardly handle this case. While some phase change models considered the molecular essence theoretically based on Herz-Knudsen equation [44], it is very difficult to provide a reliable uptake coefficient. The accuracy of interface tracking in CFD is also questionable. The second problem is the selection 
of CFD cell size. As a fundamental assumption of CFD simulation, the material must be at local equilibrium. The size of cells in HSI should be at least one magnitude larger than the mean free path. The mean free path of liquid is usually smaller than molecular diameter, but for gas, the mean free path can be several hundreds of molecular diameters. If the bubble is going to enter CFD region, the cell size has to account for both liquid and gas conditions. It causes a dilemma in choosing the right value. Assuming the gas mean free path is 100 times of molecular diameters, and the cell size is 10 times of gas mean free path, the 3-D cubic cell will contain at least $10^{9}$ molecules if it is filled with liquid. In this case, the detailed fluid dynamics are not considered, and the simulation cost of one cell is already beyond the capability of most machines. In this study, the problems are circumvented instead of being ignored. As a matter of fact, all the potential problems are induced by liquid-vapor interface. If the interface stays in MD region, the CFD approach has no problem in dealing with a single phase flow. The CFD can even have different regions and different cell sizes for the two phases separately, as long as the fluid phase in each region is already known.

\subsection{Domain re-decomposition}

In order to take full advantage of the MD approach while reducing the computational cost, the hybrid simulation needs a proper distance between the liquid-vapor interface and HSI. If the HSI is too far away, unnecessary computational effort has to be placed on simulating bulk liquid. If the HSI is too close, its fluid properties will be greatly influenced due to the phase change. Therefore, the liquid-vapor interface needs to be detected every few time steps and a follow-up re-decomposition process should take place to adjust the position of HSI according to the position of the bubble. As shown in Figure 2.4, a trigger region is defined in MD to examine the phase distribution of the fluid inside. The trigger region is at a proper distance (d1) away from CFD 
boundary so that fluid in HSI can be considered as bulk liquid. It is also at a proper distance (d2) from solid surface since the fluid near the surface has to be simulated by MD. Since the liquid and vapor densities are quite different, their interface can be easily identified by counting the molecules in each selected region. When the interface is too close to the HSI, the MD region will be extended, and when the interface is too far from HSI, the MD region shrinks to reduce the number of particles. The trigger zone is placed at a roughly constant distance away from CFD region, and it might change shape or size wherever/whenever necessary. Once a least distance between HSI and liquid-vapor interface is defined, the number of particles can be minimized if the domain is re-decomposed every time step. However, considering the errors introduced and time consumed by transferring the information, it would be better to allow the trigger zone to stay in MD region and modify the domain only if the interface is about to move out of this zone.

Figure 2.5 exhibits the possible situations when the dynamic re-decomposition method is applied. In Figure 2.5 (a), the bubble shrinks so that it is too far from the HSI. The simulation is suspended and the CFD and MD domains are reassigned. The CFD information is mapped from the original mesh to the new mesh. For the increased HSI region shown in the box, information cannot be extracted from the previous mesh. However, it can be calculated by MD simulation in the same way as P2C schemes (equation2.10 equation2.11). In addition, atoms that no longer belong to the MD domain are removed with the rest remain unchanged. In Figure 2.5 (b), the bubble grows and it is too close to the HSI. The overlap region is moved upward and new atoms will be created to fill the new region. Since the fluid in the new region has to be liquid, the number of atoms to be created is

$$
Z=\frac{\rho_{l} V}{M}
$$


where $V$ is the volume of the new region, $M$ is the atom weight. The atom creation is similar to the atom insertion for the mass flux control, except that all atoms are created at the same time. The information from CFD is used to dictate the states of these new atoms. Since the large amount of randomly inserted atoms are far from the equilibrium state, sufficient time is required for those atoms to equilibrate to a lower energy state with constraints associated with CFD states. After that, their bulk velocities are also changed to corresponding velocities in each CFD cell.

Once re-decomposition is accomplished, the simulation will be resumed. The hybrid method works again like a fixed decomposition method, until the bubble interface changes too much that another re-decomposition process is required.

For the MD domain in boiling process, it should be noticed that only the fluid close to the wall and HSI is liquid despite the expansion. The majority of the fluid after phase change is in vapor state, which has a much lower density. Therefore, the total number of atoms is expected to be only slightly changed. Since the computational cost of MD mainly depends on the number of atoms, it is believed that simulation of vapor phase is much cheaper than that of condense liquid. 


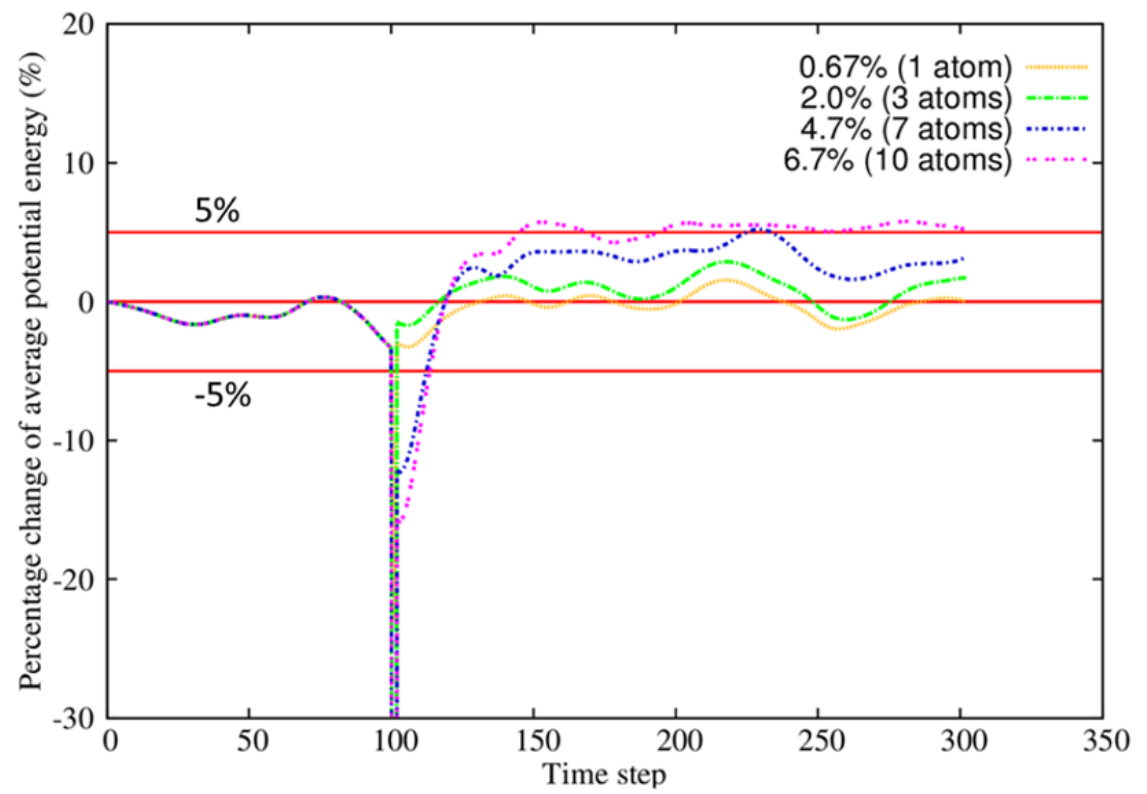

(a)

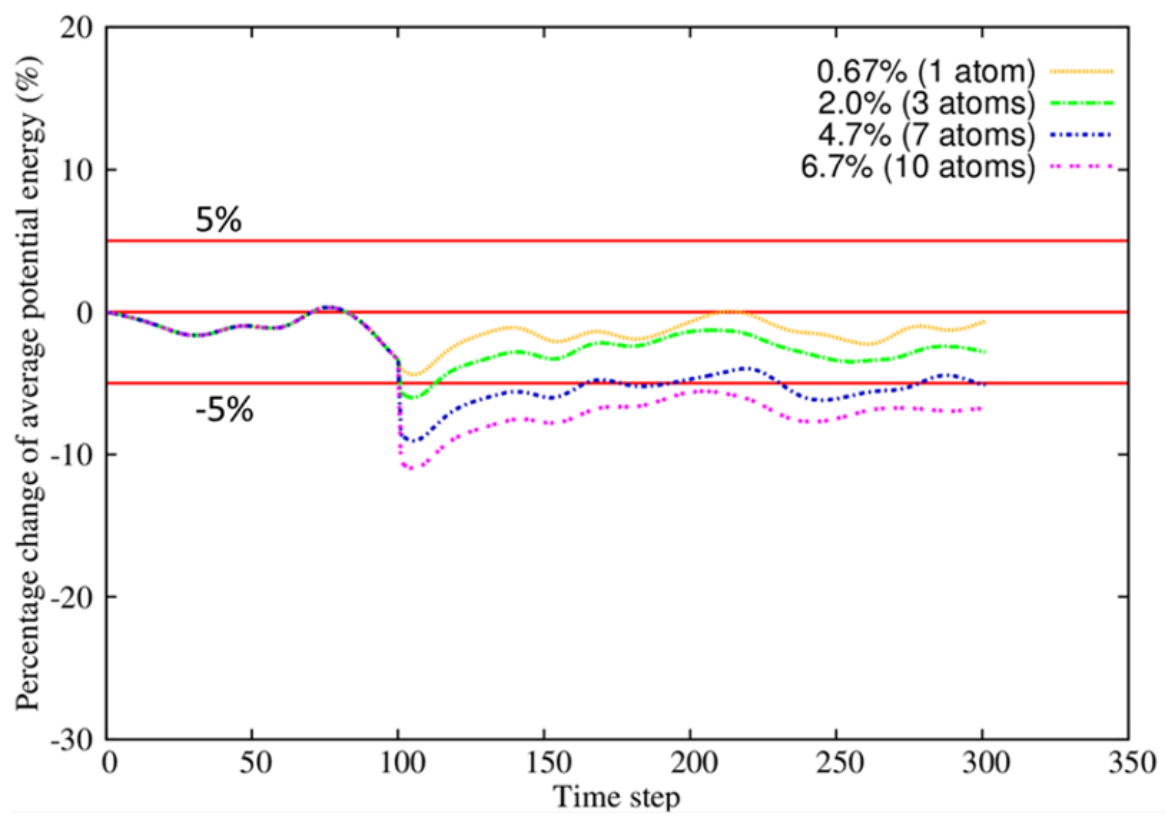

(b)

Figure 2.3: Variation of the average potential energy of argon atoms. The change of the atoms number occurs at the 100th time step. (a) Atoms are inserted. (b) Atoms are deleted 


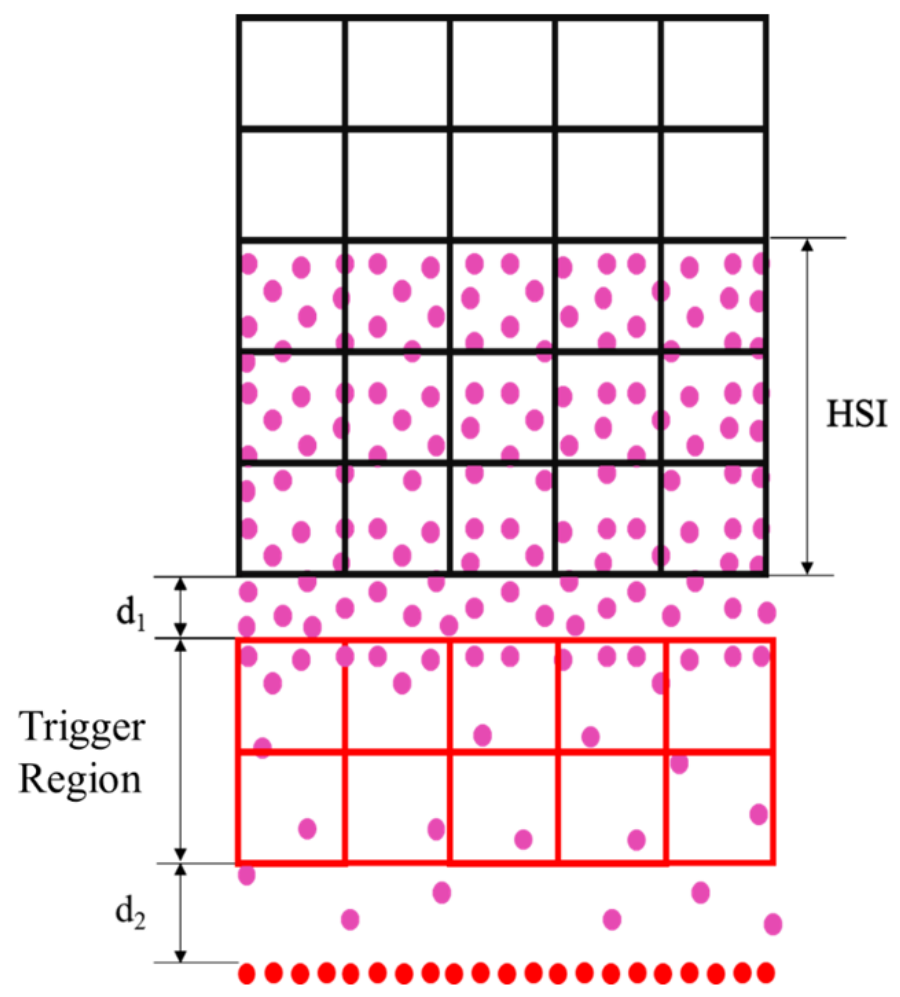

Figure 2.4: Hybrid computational domain with a trigger region. 

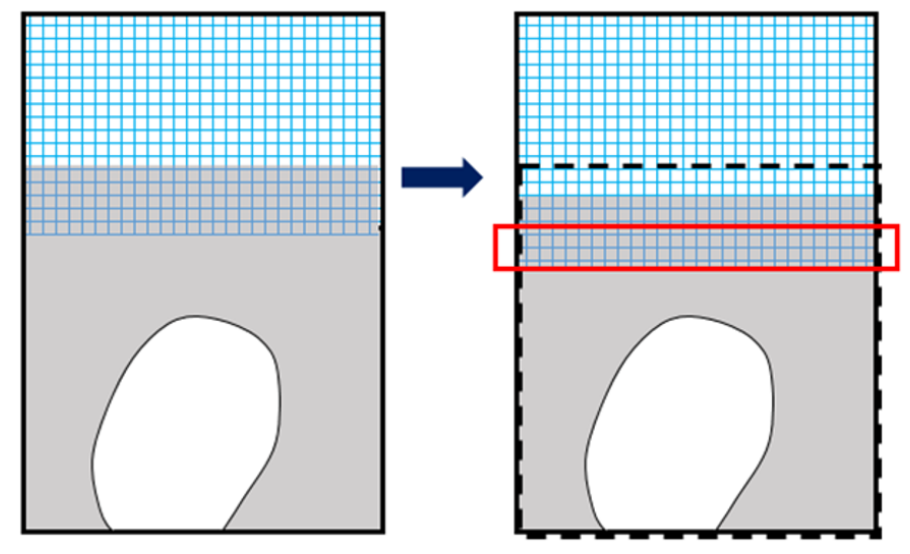

(a)
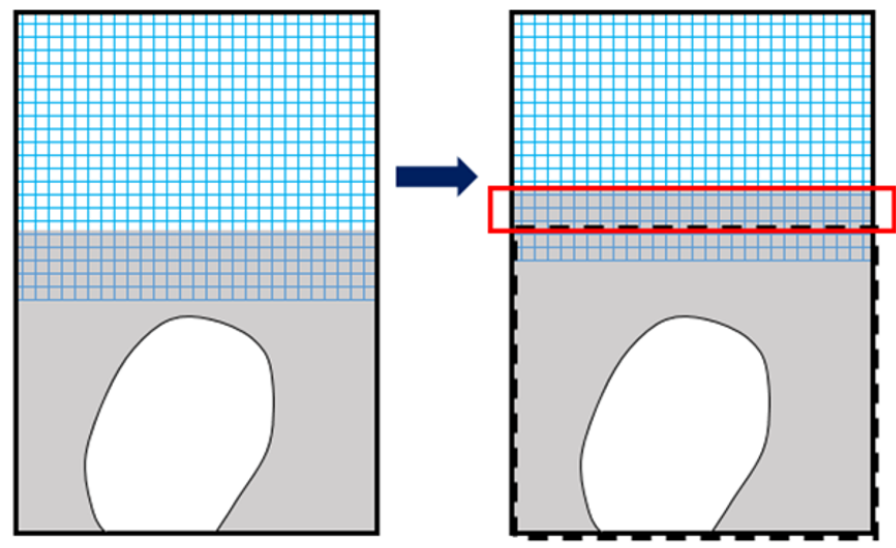

(b)

Figure 2.5: Domain re-decomposition at different conditions. (a) The bubble is too far from the HSI (b) The bubble is too close to the HSI. The dash line is the original MD domain. 


\section{Chapter 3}

\section{RESULTS}

\subsection{Model Validation}

\subsubsection{Sudden Start Couette Flow}

A simulation of sudden start Couette flow with heat transfer is used to examine the capability of the proposed schemes. As shown in Figure 3.1, a 2D computational domain with $20 \mathrm{~nm}$ in width and $26 \mathrm{~nm}$ in height is prepared. The periodic boundary condition is applied to the left and right sides. The domain from the bottom to 16 $n m$ is the MD region, which contains 11,778 fluid argon atoms. It is bounded by a fictitious top wall that imposes a perpendicular force to prevent atoms from drifting away. The potential is also governed by equation 2.3, where $r$ is defined as the distance between the atom and the wall, with $\sigma=3.405 \AA$ and $\epsilon=0.0303 \mathrm{eV}$. The bottom wall is composed of two layers of copper atoms, the top layer is held at $110 \mathrm{~K}$, and the atoms in bottom layer are stationary to model a semi-infinite potential of the solid wall [45]. The domain from $8 \mathrm{~nm}$ to the top is the CFD region, and this part is divided uniformly into $10 \times 9$ cells. Initially the fluid is equilibrated at $110 \mathrm{~K}$ and the 
bulk velocity is set to be $0 \mathrm{~m} / \mathrm{s}$. The bottom wall in MD is held at $110 \mathrm{~K}$ and kept stationary, while the top boundary in CFD has a lower temperature of $90 \mathrm{~K}$ and a constant velocity of $100 \mathrm{~m} / \mathrm{s}$. It should be pointed out that this problem is a classical single-phase problem, which can be easily and accurately solved by a standard CFD solver. Therefore, the full CFD result can serve as a reference result.

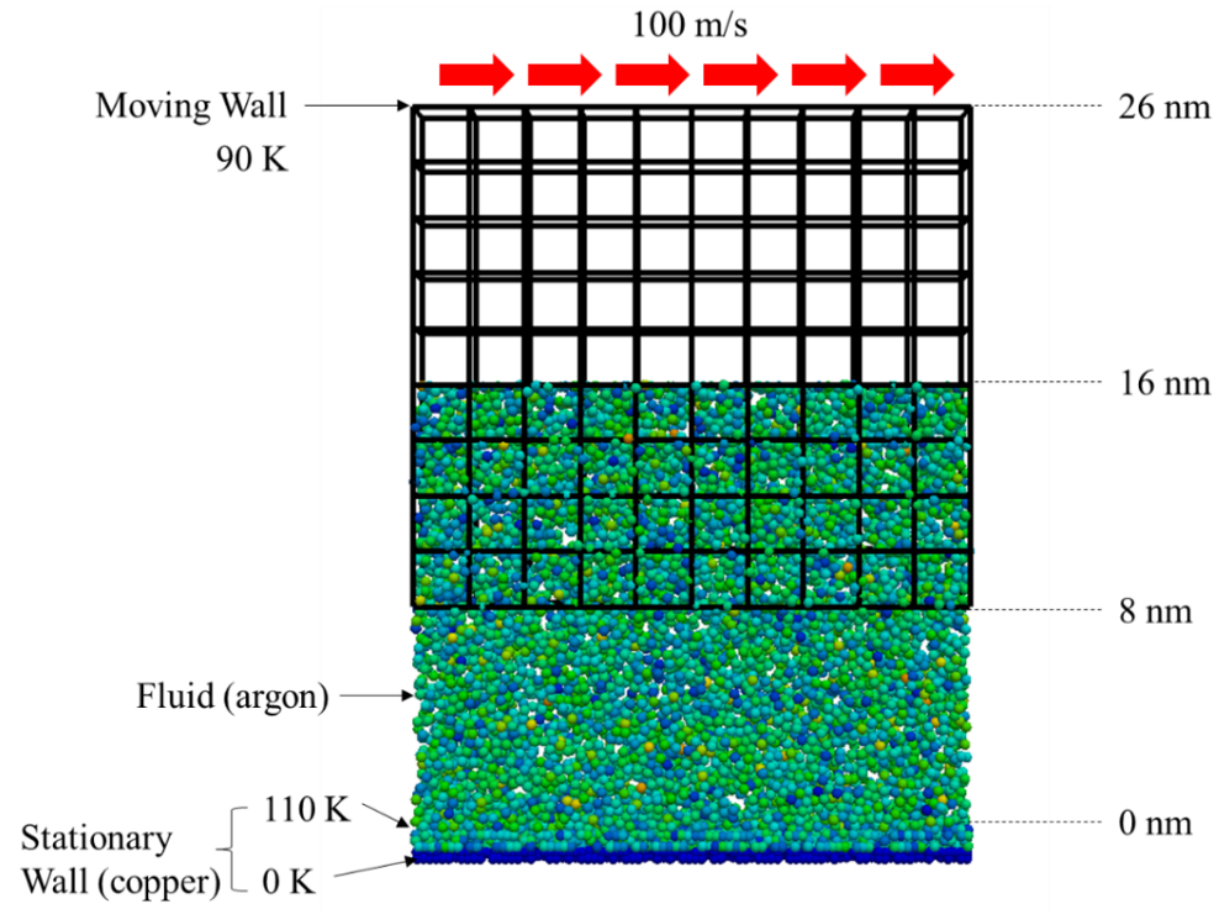

Figure 3.1: Coupling simulation domain for Couette flow with heat transfer. On the right shows the geometry height, with the liquid-solid contact surface at $0 \mathrm{~nm}$.

According to the statistical mechanics, MD simulation is always accompanied with thermal fluctuations. When the fluid properties and variables are calculated based on the simulation, the results will contain random noises. This influence can be reduced by taking a time-average of the variables, however, there are insufficient timesteps to evaluate for a transient problem. The hybrid method has the same problem since MD is involved. To minimize its influence in this problem, the hybrid simulation results are averaged over 10 cases, whose initial states are selected after different equilibration times. The state velocity and temperature profiles at the same period 
are averaged and shown in Figure 3.2(a) and Figure 3.2(b), along with the results of full CFD simulation. After the sudden change of the boundary conditions, the velocity distribution arrives at steady state at about $1.2 \mathrm{~ns}$, and for temperature it takes about 2.4 ns. The profiles show a good agreement between the coupling method and standard CFD method at all the selected time steps. It proves that the coupling schemes mentioned above are able to handle the information transfer between the two domains. However, the domain re-decomposition is not triggered due to the absence of phase change. Therefore, the method functions like a fixed decomposition hybrid scheme.
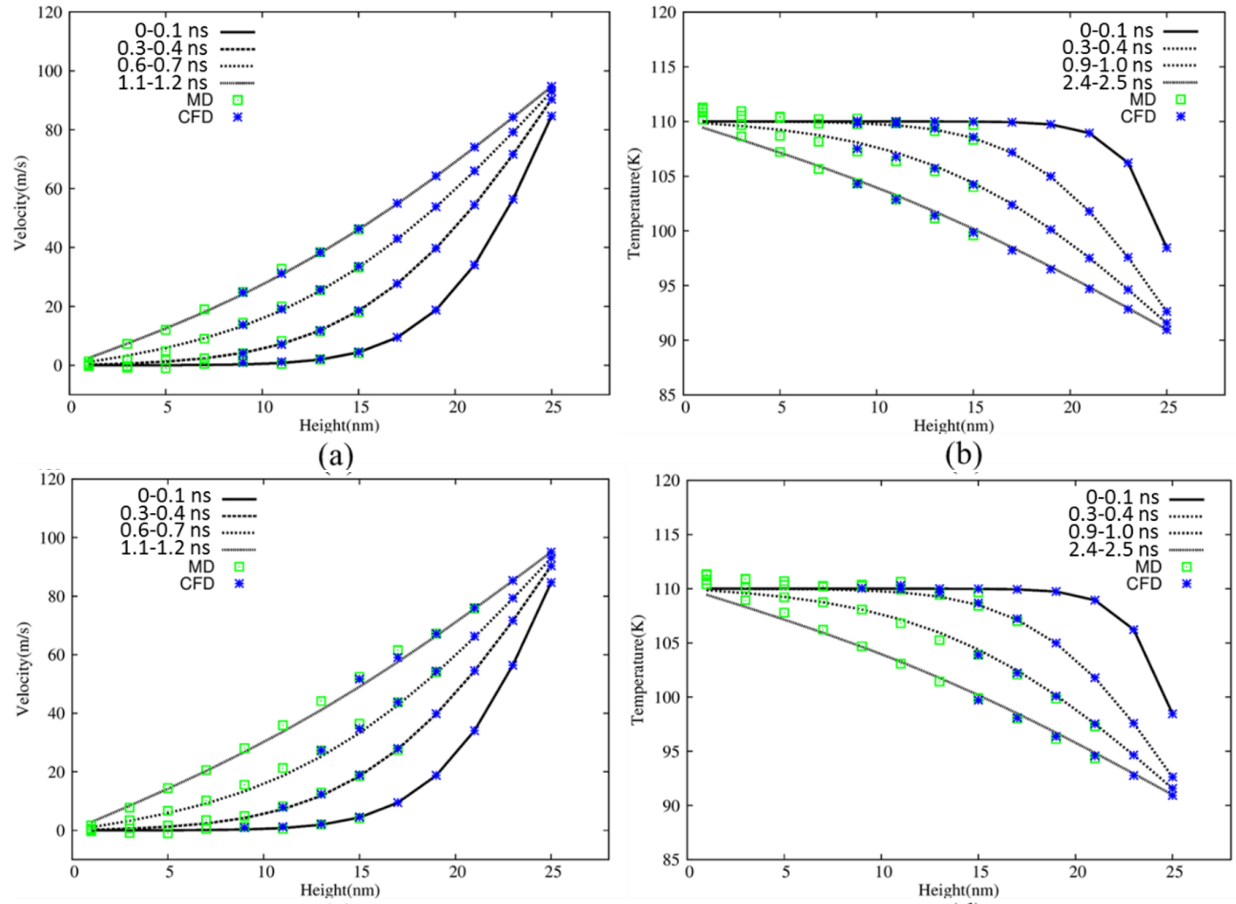

(b)

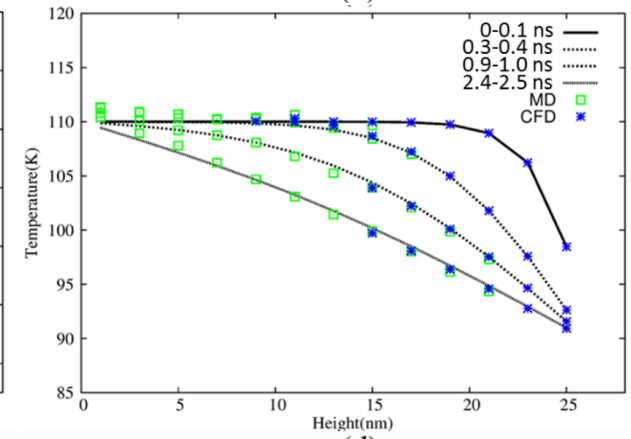

(c)

(d)

Figure 3.2: Comparison of pure CFD simulation and hybrid simulation results. Pure CFD results are shown in lines. The points are MD and CFD results in coupling method. (a) Velocity profile for simulation without re-decomposition (b) temperature profile for simulation without re-decomposition (c) velocity profile for simulation with re-decomposition $(d)$ temperature profile for simulation with re-decomposition 


\subsubsection{Couette Flow Simulation with Domain Re-decomposition}

In order to examine the domain re-decomposition process, the same fluid problem is simulated with the same setup. But this time the re-decomposition is artificially triggered at the following times: $0.21 \mathrm{~ns}, 0.41 \mathrm{~ns}$ and $0.81 \mathrm{~ns}$. The simulation domains at different times are shown in Figure 3.3. At each time, the HSI is moved upward by $2 \mathrm{~nm}$. Correspondingly, new atoms are inserted into the system while CFD region retreats at the same rate. The results of coupling simulation with re-decompositions are also averaged over 10 cases. Figure 3.2(c) and Figure 3.2(d) show the comparison of these cases with full CFD simulation case. In general, it is found that the hybrid method can still reproduce the results provided by the standard CFD solver. In addition, it is observed that the CFD data points decrease as MD data points increase at the same pace due to the change of each region size. It is also noticed that the combined domain remains unchanged all the time. In fact, it cannot be extended during the simulation, since there is no information available for a newly created region.

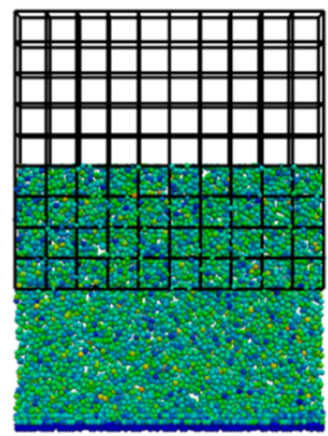

$0 \mathrm{~ns}$

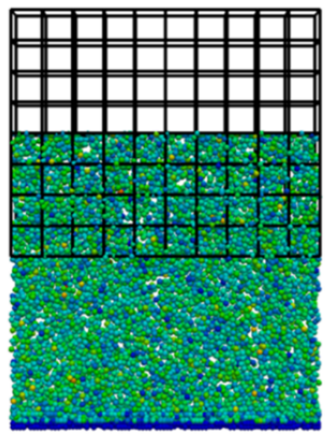

$0.21 \mathrm{~ns}$

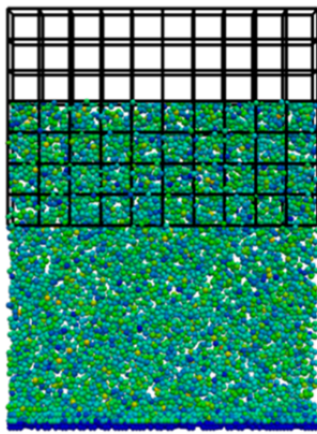

$0.41 \mathrm{~ns}$

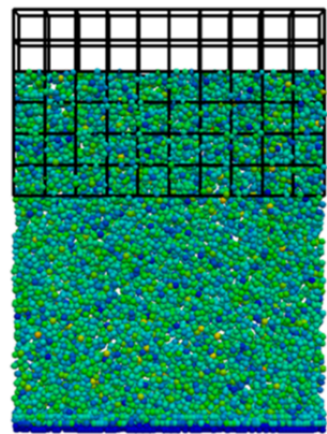

$0.81 \mathrm{~ns}$

Figure 3.3: Coupling simulation domains at different times. The re-decomposition times are $0.21 \mathrm{~ns}, 0.41 \mathrm{~ns}$ and $0.81 \mathrm{~ns}$

In the studies above, the hybrid method is examined to be capable of solving a transient Couette flow. The results from the combined domain match well with 
the results from full CFD simulation. In addition, the re-decomposition strategy is applied to the same problem, and it is proved to have minimal effect on the simulation.

Based on these facts, the simulation can be modified as a vapor bubble nucleates in the MD domain, while the functions of HSI and CFD domain remain unchanged. As long as the bubble is limited within MD region, all multiphase physics can be considered intrinsically. Therefore, the hybrid method can be used to solve the specified phase change problem without further assumptions.

\subsection{Nucleate Boiling on Structured Surface}

In the following, a nucleate boiling simulation is performed with the dynamic redecomposition coupling method. As shown in Figure 3.4, a solid surface with nanostructure is created with atoms. The MD region has a height of $55 \mathrm{~nm}$ and a width of $50 \mathrm{~nm}$, and the cavity size is $5 \mathrm{~nm} \times 5 \mathrm{~nm}$. The top boundary of CFD is placed sufficiently far away from the solid wall such that the boundary effect could be reduced as much as possible. The dynamic HSI introduced above is used to connect these two regions. The system has an open boundary at the top where a constant pressure of $1 \mathrm{MPa}$ is applied. The bottom of MD region is bounded by copper atoms. Similar to the previous case, one layer of stationary atoms shapes the wall geometry, and another layer of atoms on their top are free to move. The left and right boundaries remain periodic. At $1 \mathrm{MPa}$, argon has a boiling point of $116 \mathrm{~K}$, the simulation conditions are designed based on this fluid property. At the beginning, the fluid is equilibrated at a temperature close to but below boiling point, then a modest superheat is applied to the solid surface to induce the nucleate boiling. Since the fluid temperature is around the boiling point before and after heating, all liquid properties used in coupling schemes and CFD are considered to be unchanged and equal to those for saturated liquid at $1 \mathrm{MPa}$ and $116 \mathrm{~K}$, as displayed in Table 3.1. The vapor 
properties are not used since the HSI and CFD regions only accounts for liquid.

\begin{tabular}{|r|l|}
\hline Density $\left(K g / m^{3}\right)$ & 1196 \\
\hline$C_{p}(J / g K)$ & 1.2771 \\
\hline Dynamic Viscosity $(\mu P a s)$ & 120.82 \\
\hline Thermal Conductivity $(W / m K)$ & 0.08828 \\
\hline
\end{tabular}

Table 3.1: Argon fluid properties at $116 \mathrm{~K}, 1 \mathrm{MPa}$

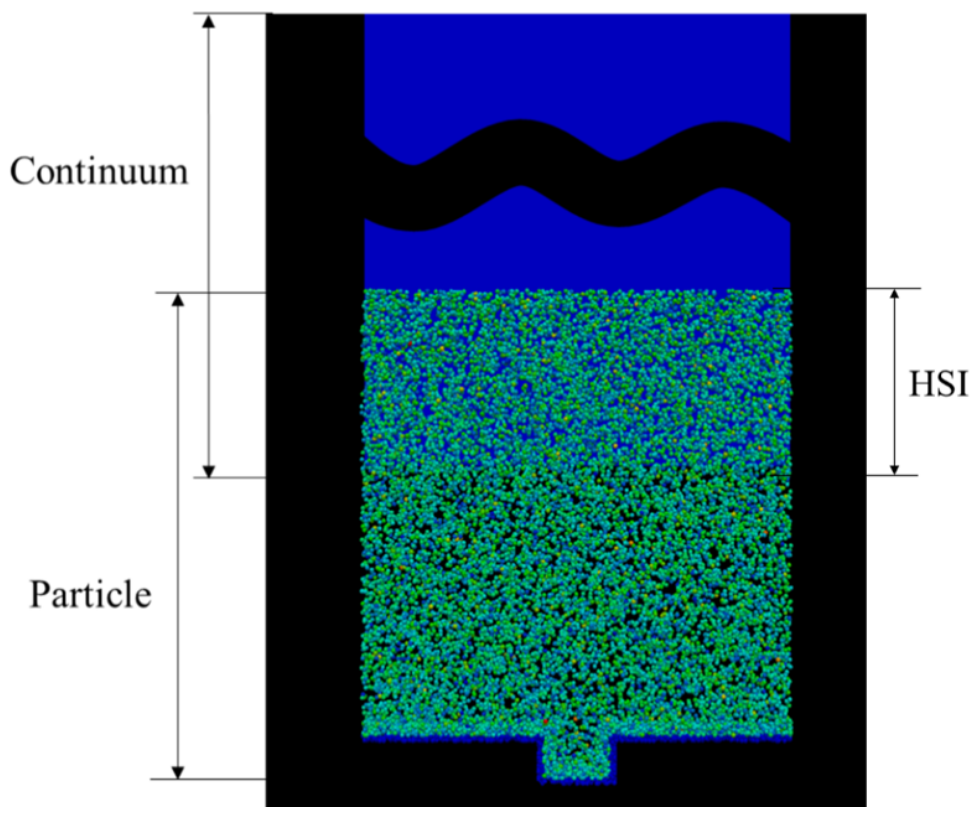

Figure 3.4: The geometry of the hybrid simulation case for nucleate boiling

Before performing coupling simulation, the argon atoms are created in MD domain and their density is adjusted to match the liquid density. The fluid is carefully equilibrated at $110 \mathrm{~K}$ with negligible bulk velocity. The potential energy of atoms is shown in Figure 3.5, the copper atoms are not included. At steady state, the potential energy distribution in internal fluid is homogeneous, while the fluid adjacent to the solid surface has lower potential energy due to the interaction between copper and argon atoms. After equilibration, all the thermostats on the fluid are removed, the solid atoms temperature is suddenly changed to $130 \mathrm{~K}$ and it is maintained by exterior energy flux. With the heat conduction through the surface, fluid atoms near the solid wall are heated up, leading to a fierce motion. Meanwhile, part of the energy from 
heating is transferred into potential energy, as shown in Figure 3.5(b). The random distribution of the energy appears to be disturbed by the heating.

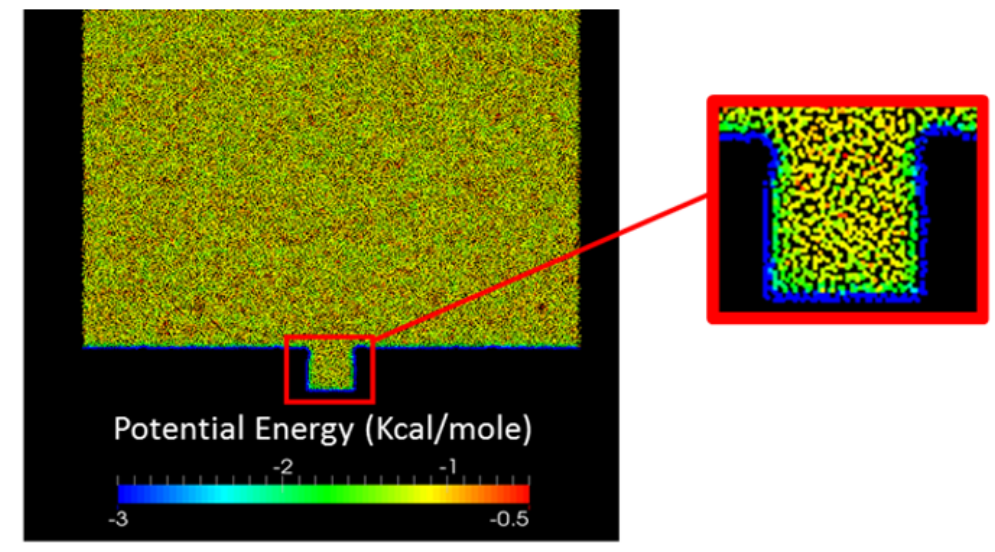

(a) $0 \mathrm{~ns}$

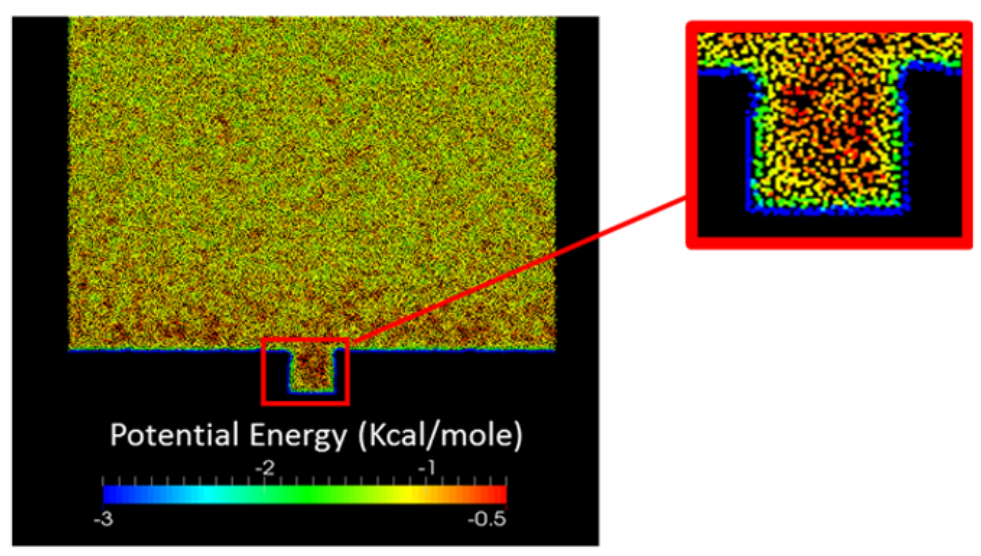

(b) $1.1 \mathrm{~ns}$

Figure 3.5: The potential energy of argon atoms during heating. (a) Potential energy right after equilibration (b) potential energy at $1.1 \mathrm{~ns}$

Although the solid temperature is maintained above boiling point, it takes a while before the phase change taking place. In the meantime, the fluid particles gradually accumulate energy from heating, but they are not able to break away from solid atoms. In Figure 3.6, a small bubble is observed at around $1.5 \mathrm{~ns}$. It shows the cavity at the center becomes the only nucleation site. The inner fluid of the bubble has lower density and higher potential energy, thus it can be easily distinguished as vapor phase. The bubble grows quickly once it nucleates. As it approaching is HSI, 
the simulation domain is re-decomposed automatically. In order to accommodate the bubbles growing size, the MD boundary is moved upward, and the CFD region retreats to make room for HSI. This process works like the previous one, except that the re-decomposition is dictated by the interface position. The bubble has a sphere shape firstly, but later it appears to be compressed from the two sides. As a result, it grows a lot higher but not much wider. The compression is likely to be caused by the periodic boundary. As the bubble size becomes comparable to the width, the boundary effect can be significant.

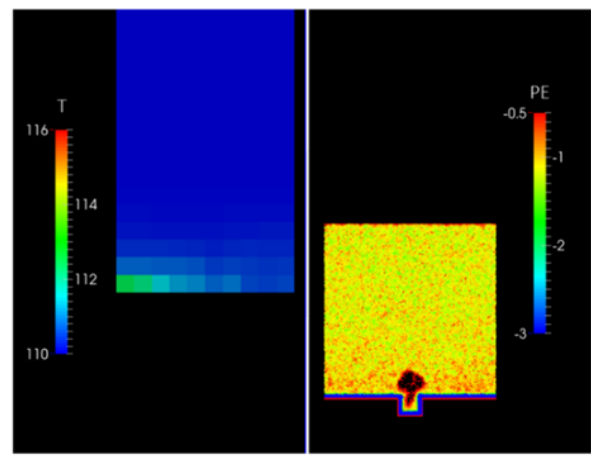

(a) $1.5 \mathrm{~ns}$

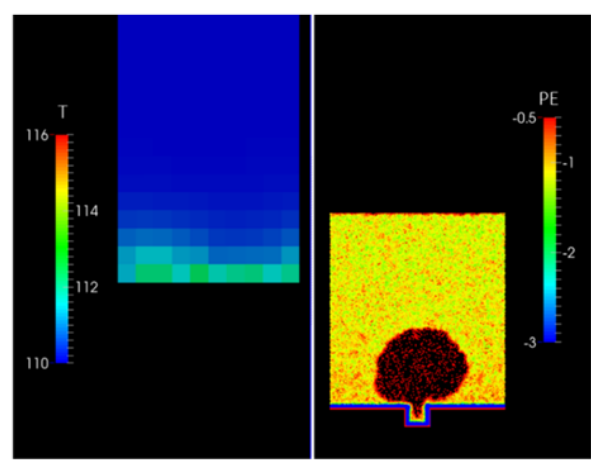

(c) $2.0 \mathrm{~ns}$

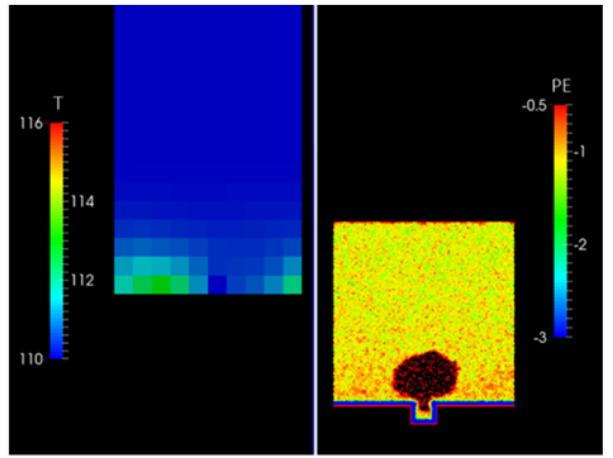

(b) $1.8 \mathrm{~ns}$

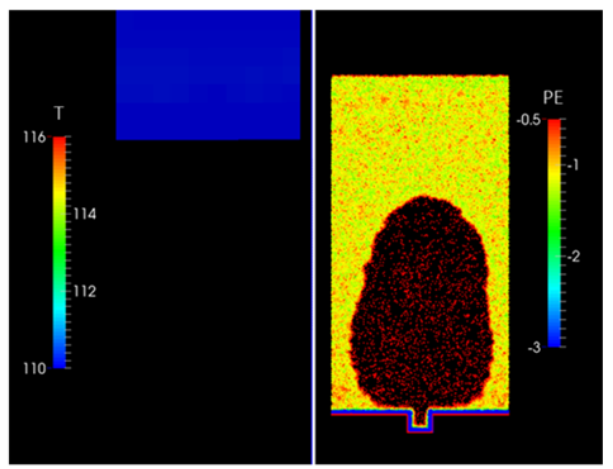

(d) $2.4 \mathrm{~ns}$

Figure 3.6: Nucleate boiling on structured surface at $130 \mathrm{~K}$. The CFD region shows the temperature distribution, and the MD region shows the atoms potential energy.

A close look at the three-phase contact region is shown in Figure 3.7, which is selected from the nucleate boiling at $2.4 \mathrm{~ns}$. The solid copper atoms are also displayed. In the MD simulation, the nanoscale discontinuity at liquid-vapor interface is considered. With the high-resolution modelling, the interface appears to be unstable 
and distorted at the initial state of bubble nucleation, which makes the effect of surface tension and line tension even more complex. It is also noticed that a layer of atoms is absorbed to the solid even though the fluid near the cavity is sufficiently heated. This region is often referred to as non-evaporating thin film region [46]. Due to the strong interaction of copper atoms, this layer can hardly escape from the surface. The tiny structures in this region show that a MD simulation is necessary when their size is comparable to the bubble size.

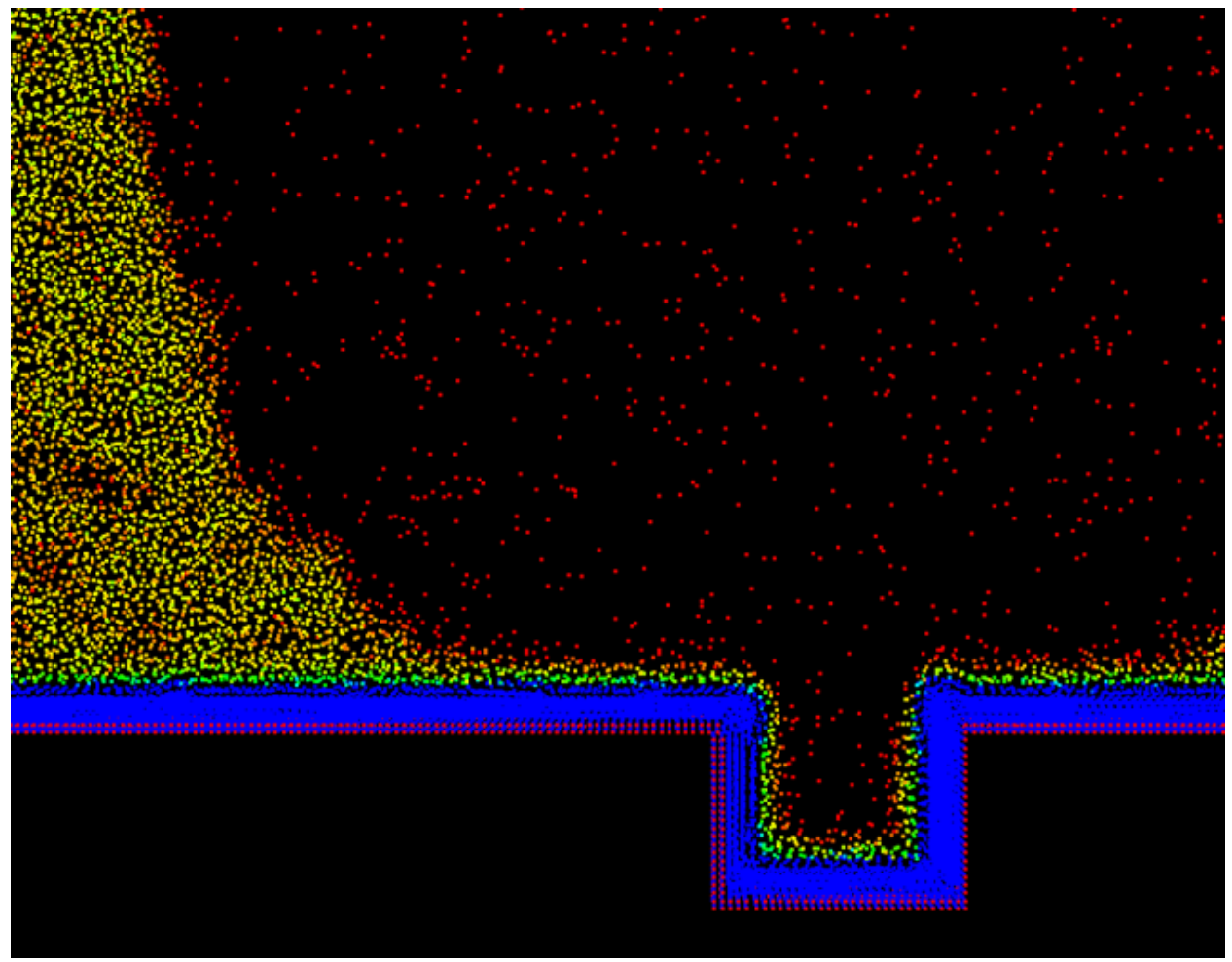

Figure 3.7: Three-phase contact region

With the same initial conditions, the boiling is simulated as the solid temperature is changed to $140 \mathrm{~K}$. This time, the phase change occurs earlier at the higher superheat, as displayed in Figure 3.8. While the first nucleation site is still at the cavity, the surrounding fluid atoms are more activated. Sometimes small bubbles are observed to emerge directly on flat surface. The vapor-solid contact line extends quickly. As a result, the meniscus region under the bubble is not clearly displayed. 
Since the simulation domain is periodic, it is finally approaching a film boiling.

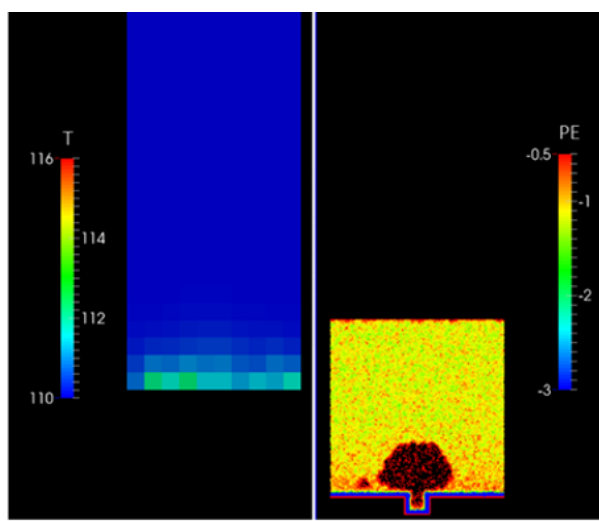

(a) $1.0 \mathrm{~ns}$

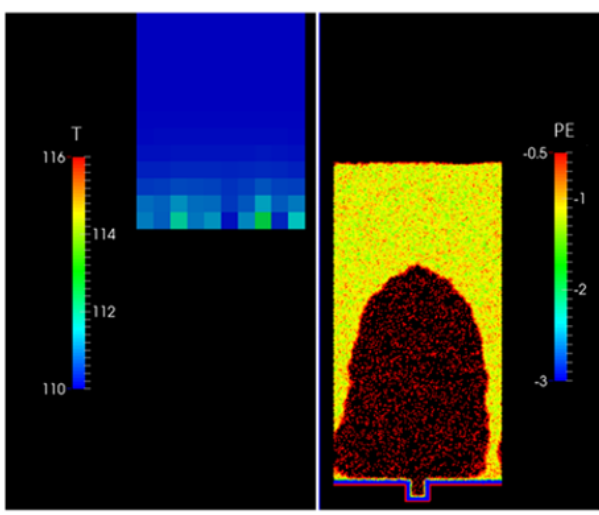

(c) 2 ns

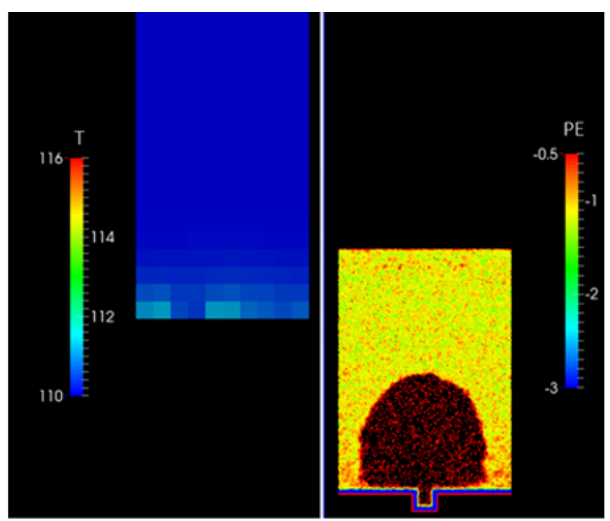

(b) $1.5 \mathrm{~ns}$

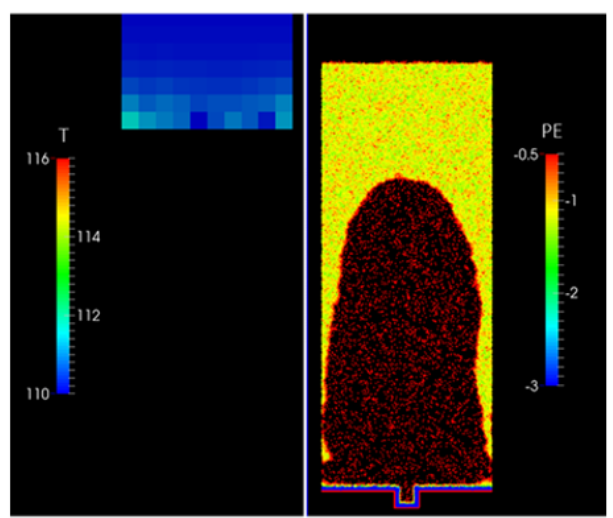

(d) $2.4 \mathrm{~ns}$

Figure 3.8: Nucleate boiling on structured surface at $140 \mathrm{~K}$. The CFD region shows the temperature distribution, and the MD region shows the atoms potential energy.

As the boiling induces a significant mass flux crossing the HSI, the number of atoms in MD region is closely related to the phase change rate and bubble dynamics. The variation with respect to time is plotted in Figure 3.9. At first, the total number of atoms decreases slowly, that is when the liquid near the solid wall expands under heating. After a while, phase change happens and large amounts of molecules are pushed out of the MD region. The bubble keeps growing and when the domain redecomposition is performed, a new layer of liquid molecules is inserted. The atoms number increases instantly, and later it gradually decreases as evaporation going on. The same pattern is observed again once the bubble is too close to the new HSI. There is no data point on the jumps, since the time for atoms insertion and 
equilibration is not counted into simulation time. At this point, it is worth examining

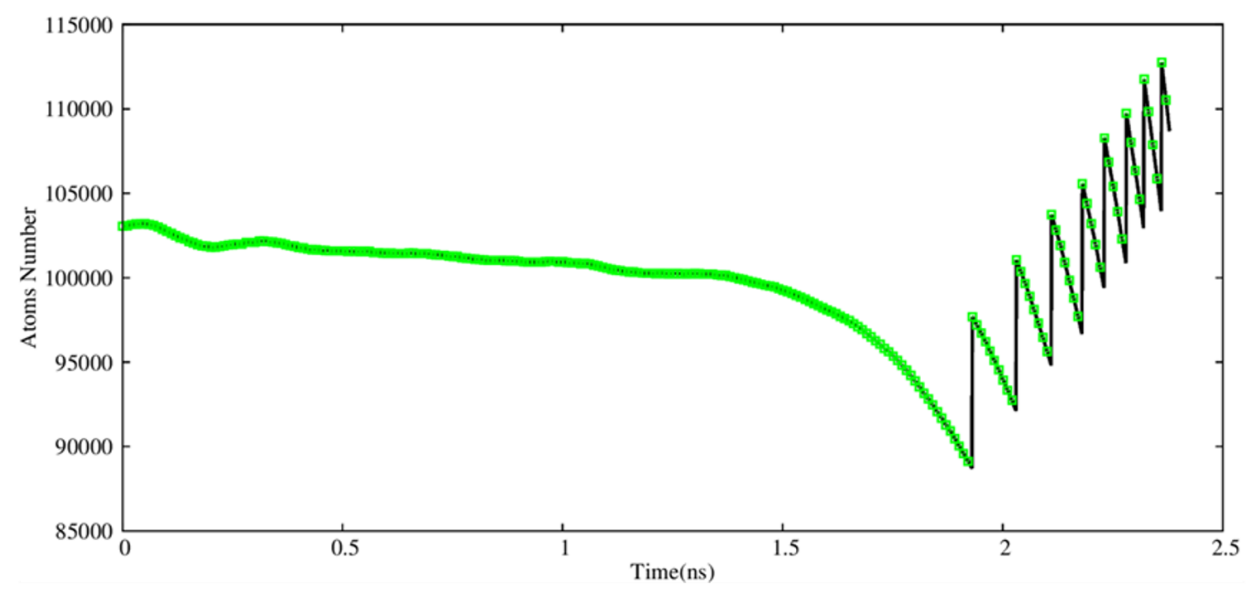

(a)

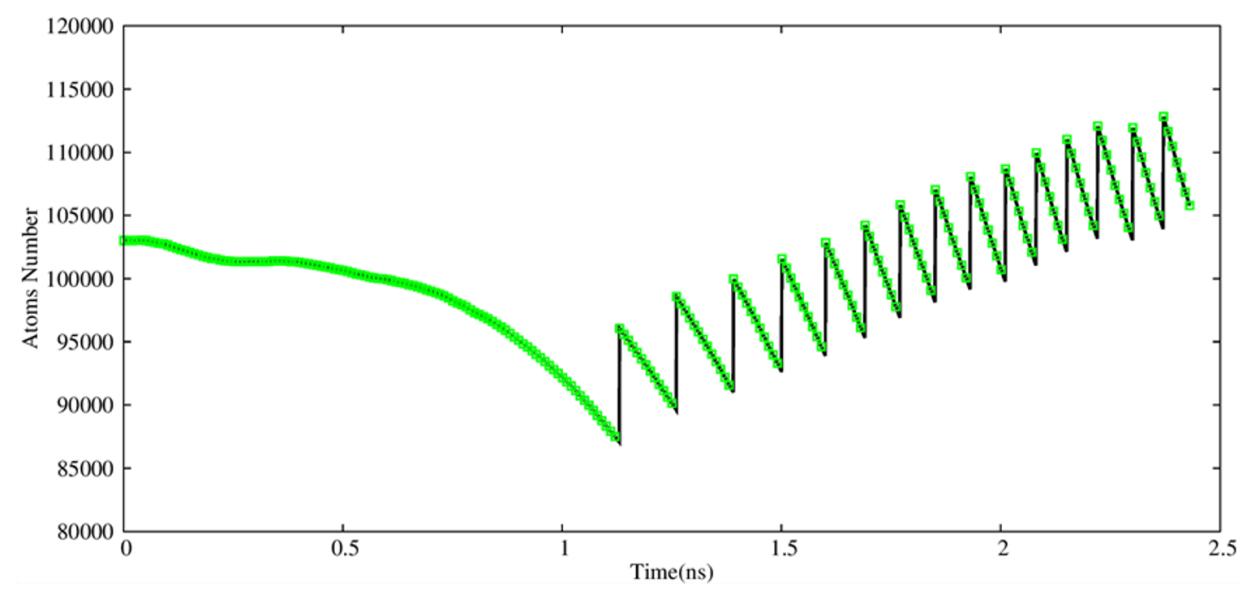

(b)

Figure 3.9: Number of atoms variation over time. (a) Wall temperature is $130 \mathrm{~K}$ (b) Wall temperature is $140 \mathrm{~K}$

how much the domain re-decomposition method has reduced the computational cost. As many studies point out, the hybrid simulation method has an advantage over pure MD method when it comes to computational efficiency. The main reason is that the number of particles in system can be reduced a lot. Nevertheless, most of the computing resources are still consumed on MD simulation. In this study, the particle number is monitored to represent the computational load. For the case in Figure 3.6, the variation of atoms number is plotted with line and points. Assuming the same problem is solved by a fixed-decomposition method, the atoms number change 
can be estimated based on the known results. The comparison of two conditions is shown in Figure 3.10. Figure 3.10(b) shows the case of a fixed-decomposition
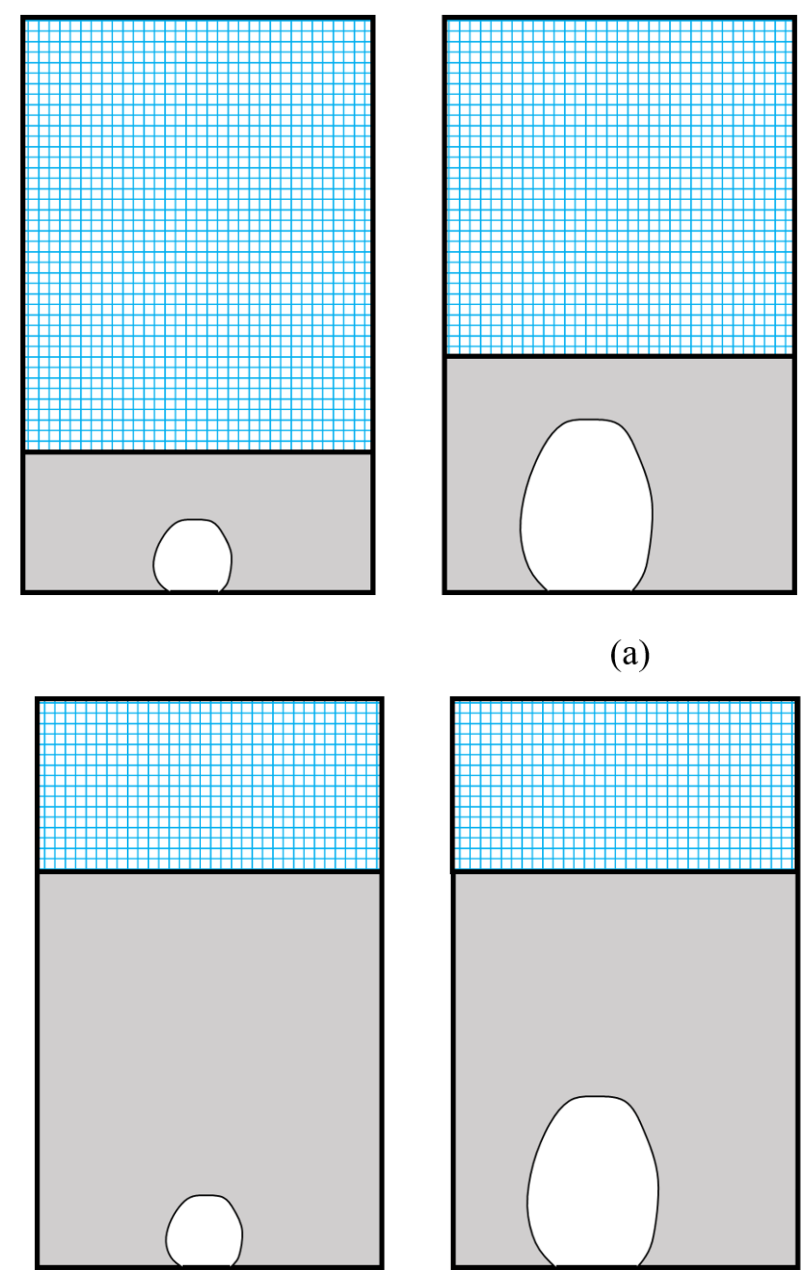

(b)
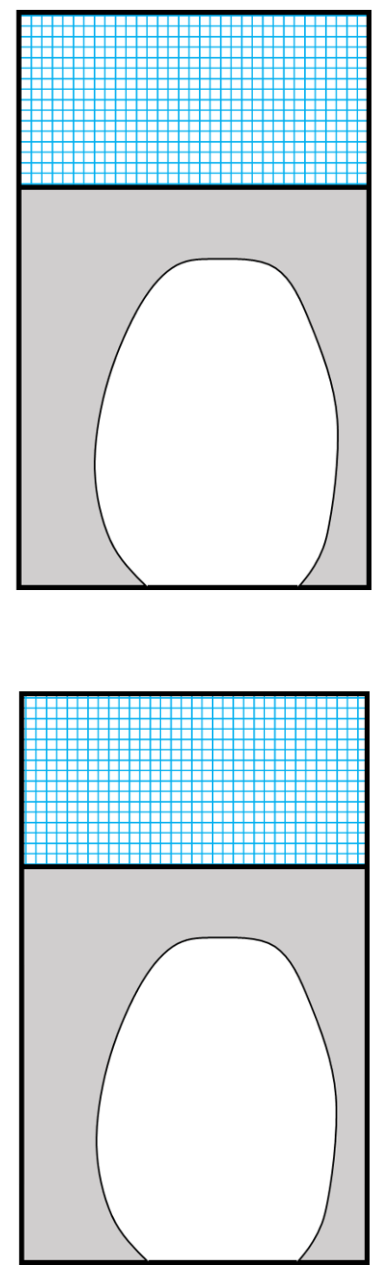

Figure 3.10: CFD and MD domains at different time steps

method, CFD will only serve as an open boundary. As the bubble needs to be confined in MD region, the CFD region needs to be set far enough so that the bubble can never touch it. Under this condition, the initial MD region should be at least the maximum size in the dynamic re-decomposition method. The domain is decomposed into MD and CFD regions at the beginning, and remain unchanged throughout the simulation. Therefore, the initial number of atoms can be estimated by the liquid density and MD domain size, while the final number of atoms should be the same 
as it is in the dynamic re-decomposition simulation. Since the phase change rate should not be changed by changing the method, the atoms number variation for a fixed decomposition method can also be estimated, as shown in Figure 3.11. The graph shows the number of atoms in a fixed decomposition method is much larger in most of the time. Obviously, the initial atoms number has to be further increased if the bubble finally grows larger. Therefore, for an extended domain with longer simulation time, the dynamic re-decomposition method has a huge advantage over a fixed decomposition method.

Figure 3.12 shows the cumulative energy added to the copper layer to keep it at constant temperature. In general, the energy flux is higher for the case with larger superheat. Since the bottom boundary in the coupling simulation is adiabatic, the energy is mostly absorbed by the fluid atoms, and transferred into kinetic or potential energy. When the solid temperature is changed at the beginning, the energy input increases sharply. The heat transfer soon slows down, as the fluid adjacent to the surface is heated. It is also observed that significant energy flux enters the fluid before the phase change occurs, as the atoms near the wall keep gaining internal energy. Though the bubble nucleation in the two cases happens at different times, it is interesting to notice that the total energy absorbed by the fluid before phase change is very close. After the bubble emerges, the total energy flux keeps increasing to support the continuous phase change during bubble growth. Due to the limited size of computational domain, the simulation turns into film boiling once the bubble approaches the periodic boundaries. Therefore, only the onsets of nucleate boiling at molecular level are successfully displayed. The same method can be easily developed for a 3-dimensional problem with more complex structured surface if a higher computational capability is provided. 


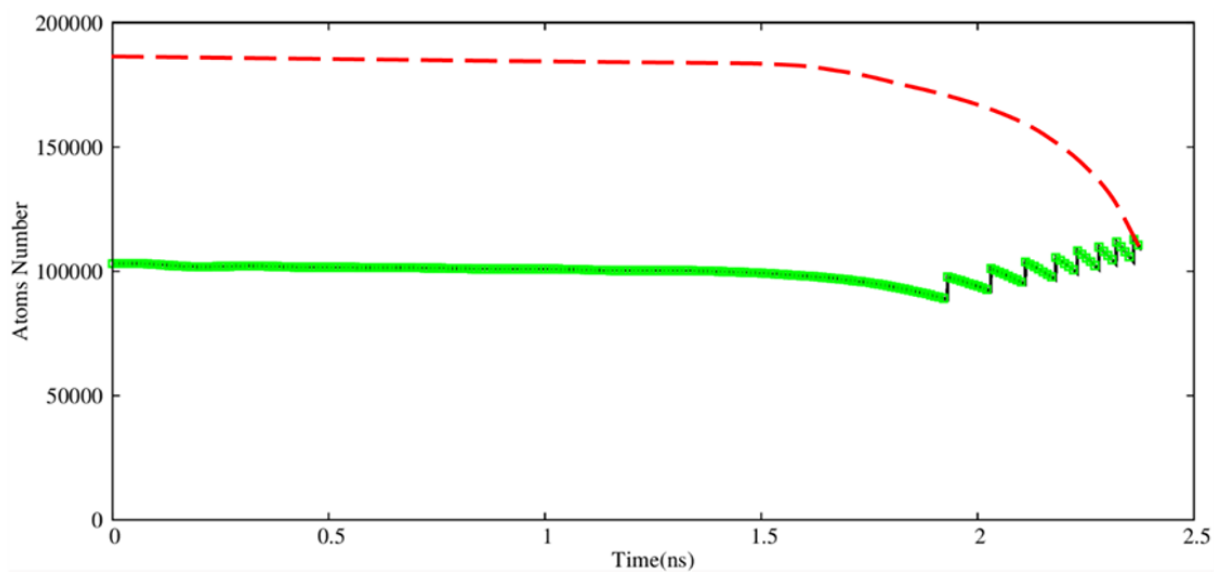

Fixed Decomposition - - Dynamic Re-decomposition

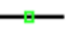

(a)

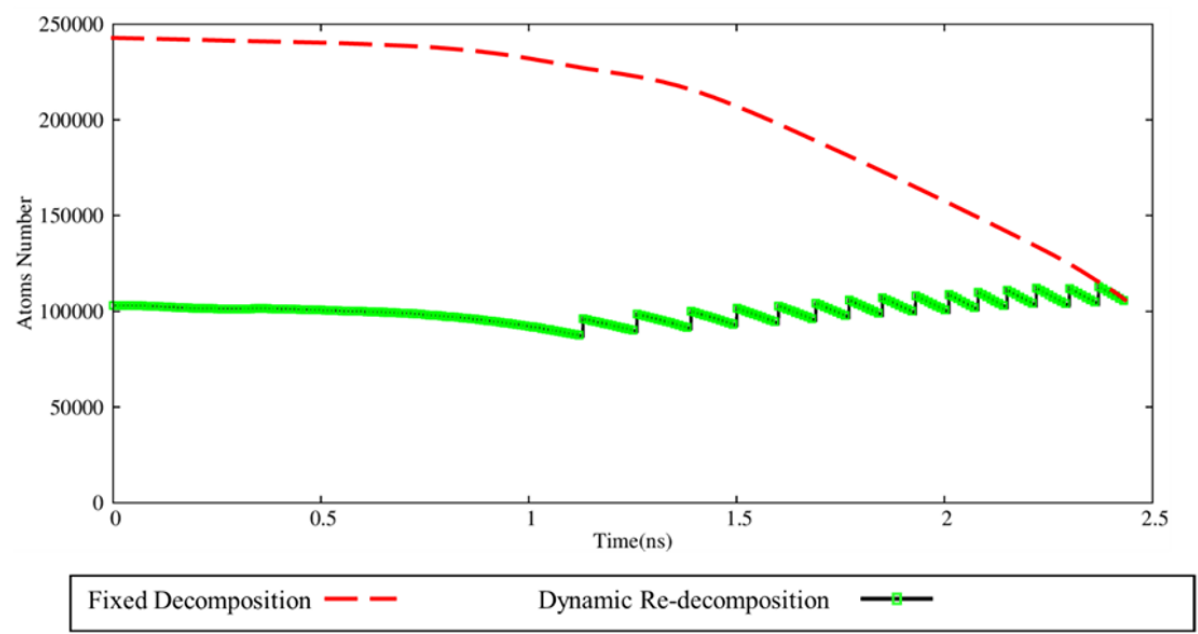

(b)

Figure 3.11: Comparison of atoms number in simulation. The dash line show the estimated atoms change if a fixed decomposition method is used for the same case. (a) Wall temperature is $130 \mathrm{~K}$ (b) Wall temperature is $140 \mathrm{~K}$ 


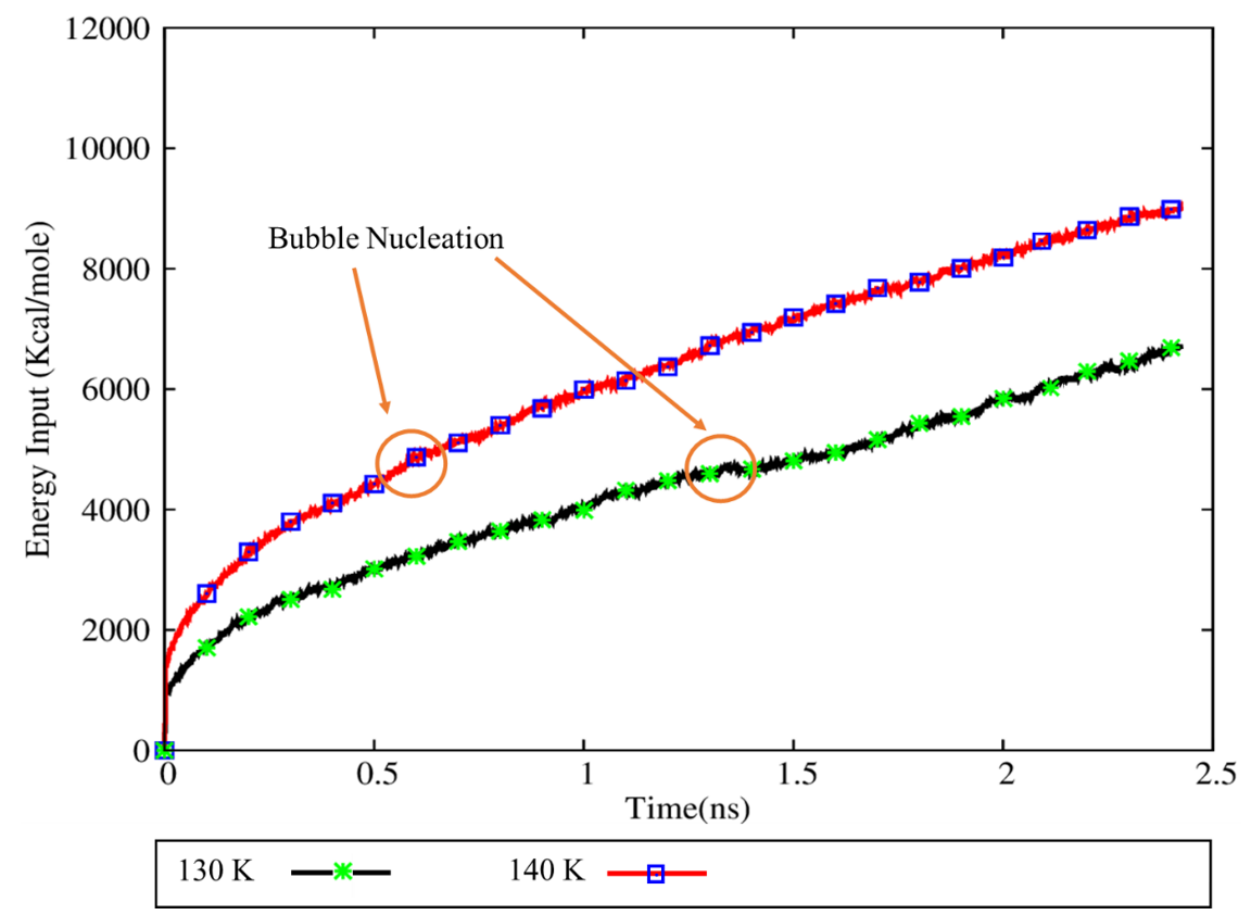

Figure 3.12: The cumulative energy flux flowing into the system 


\section{Chapter 4}

\section{CONCLUSION AND DISCUSSION}

A dynamic re-decomposition hybrid atomistic-continuum method that extends the capability of previous coupling method by allowing the HSI to track the discontinuous regions is presented. It is demonstrated that the current coupling approach is capable of reproducing Couette flow results that is also solved through full CFD method. In addition, the method is applied to simulate nucleate boiling phenomena on nanostructured surface. The simulation reveals atomic level behaviors of fluids nearby the solid and the variation of energy flowing into fluid during this process. The efficiency of the dynamic re-decomposition method is also examined through comparing to the fixed decomposition method. It is found that the dynamic re-decomposition method needs less atoms to simulate the problem with the same computational domain. The approach is promising for solving micro/nanso-scale boiling problems where nano-bubble dynamics, phase change rate, and wettability effects are still not well understood.

For all the cases described above, the shape of HSI maintains flat all the time. This is not very computationally efficient when the simulation domain is much wider than the bubble. In our future work, the dynamic re-decomposition method will be 
further studied with respect to complex geometry. An improved method needs more consideration on determining the location/shape of boundaries thereafter the HSI, as shown in the Figure 4.1. The geometry of HSI could be in an arbitrary shape, which depends on the shape of liquid-vapor interface in the MD region whose domain intends to be minimized. However, to the knowledge of the authors, the condition could be even more complicated if multiple bubbles emerge and detach during the process. It should also be noted that it is unnecessary to find a perfect shape of HSI that surrounds the bubbles, since the over-sharpness between two adjacent bubbles could cause numerical instability. Therefore, a satisfactory boundary depends on the trade-off between computational cost and mesh quality. It is also indicated in the Figure 4.1 that a CFD domain could also be placed within the vapor domain once the bubble is extremely large. Though it is true that the simulation cost on vapor is usually negligible, if the size of vapor region is thousands of times larger than that of liquid region, the bulk vapor should be simulated through a separate CFD region where another HSI should be utilized to couple its outer MD region. In this way, the only regions left in MD are the walls and liquid-vapor interfaces where the discontinuity and fluctuations play a significant role.

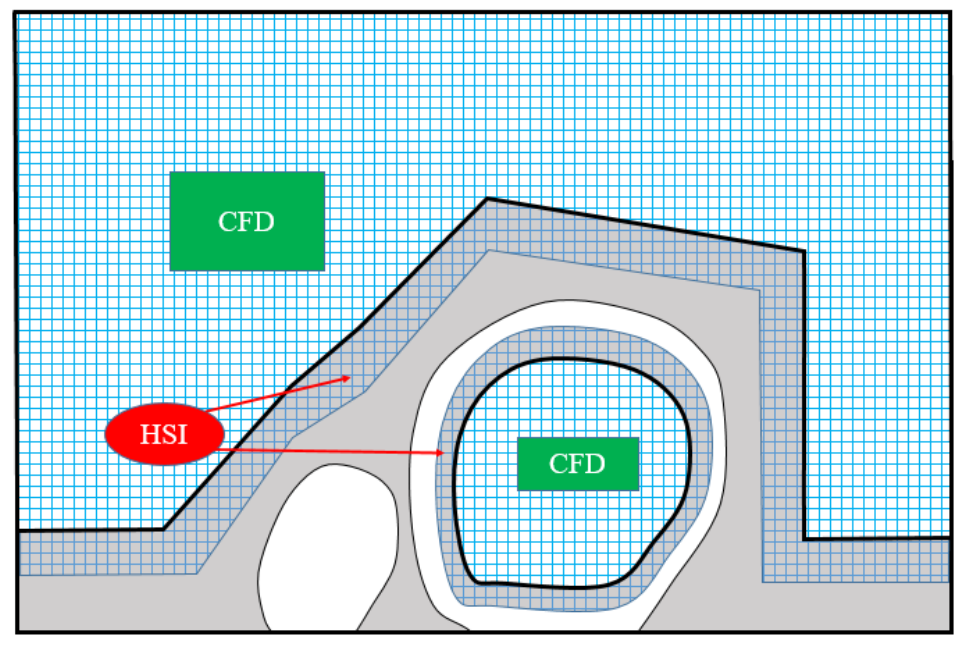

Figure 4.1: Domain decomposition when multiple bubbles exist 


\section{Appendix A}

\section{Coupling Loop}

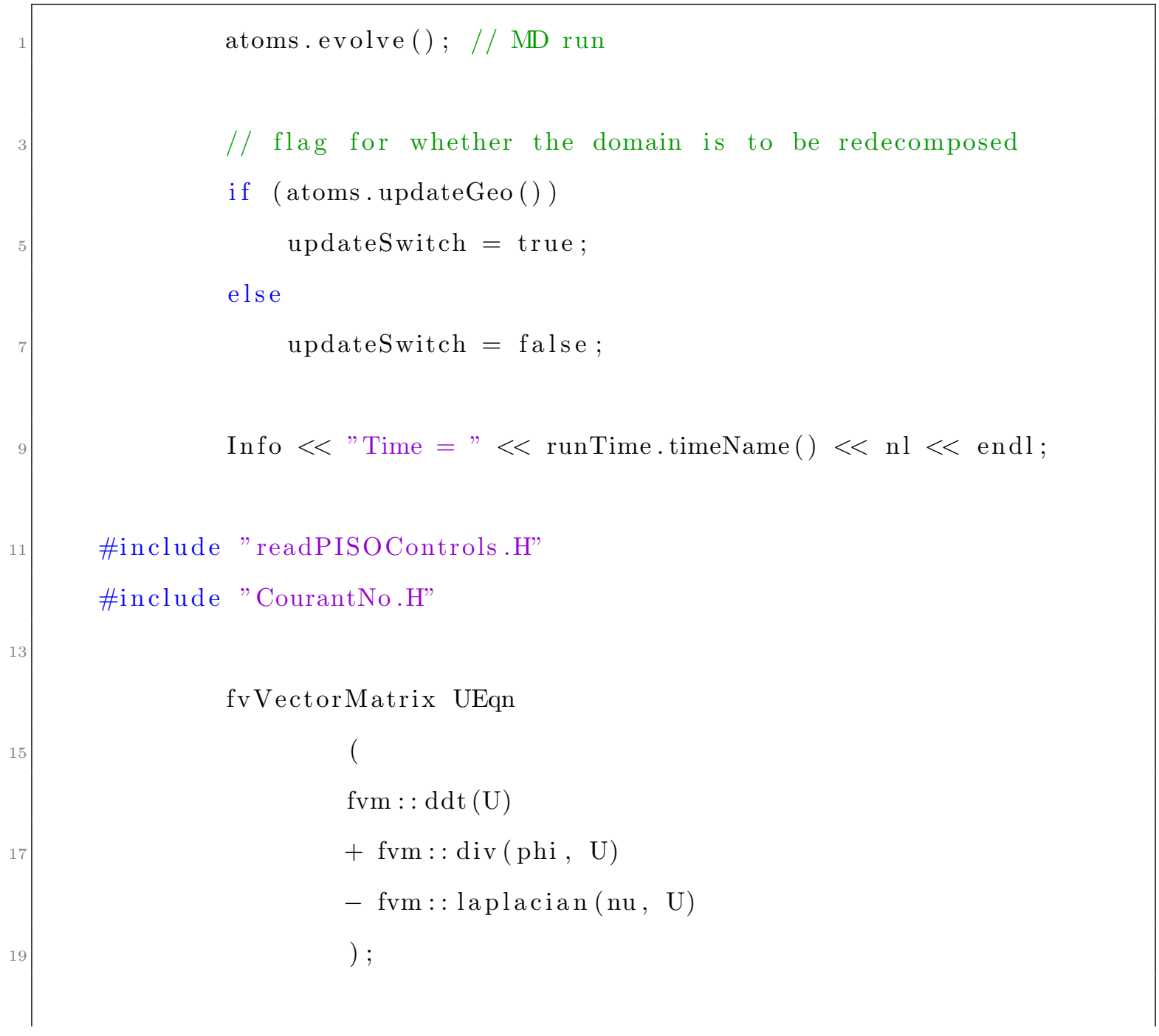




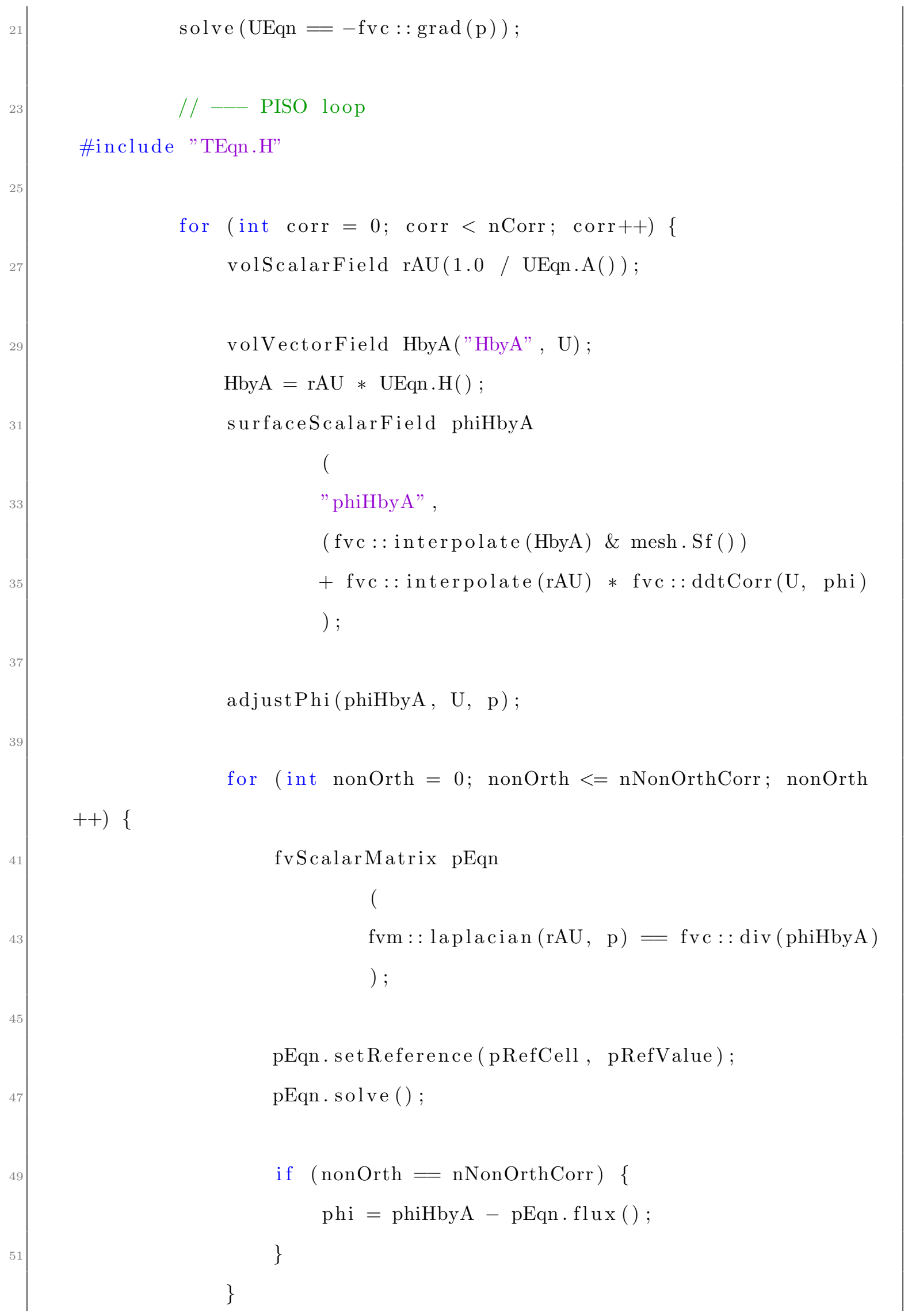



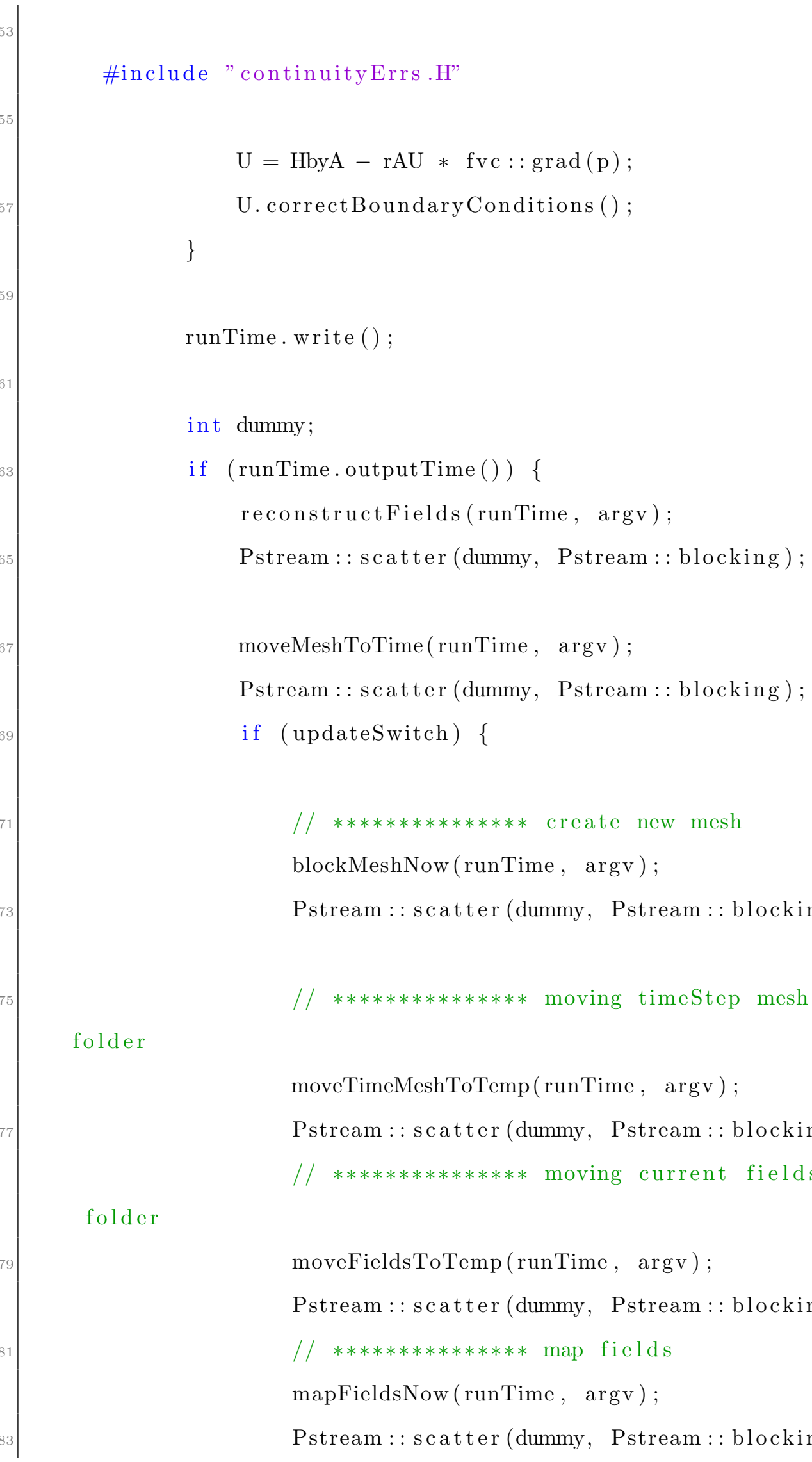


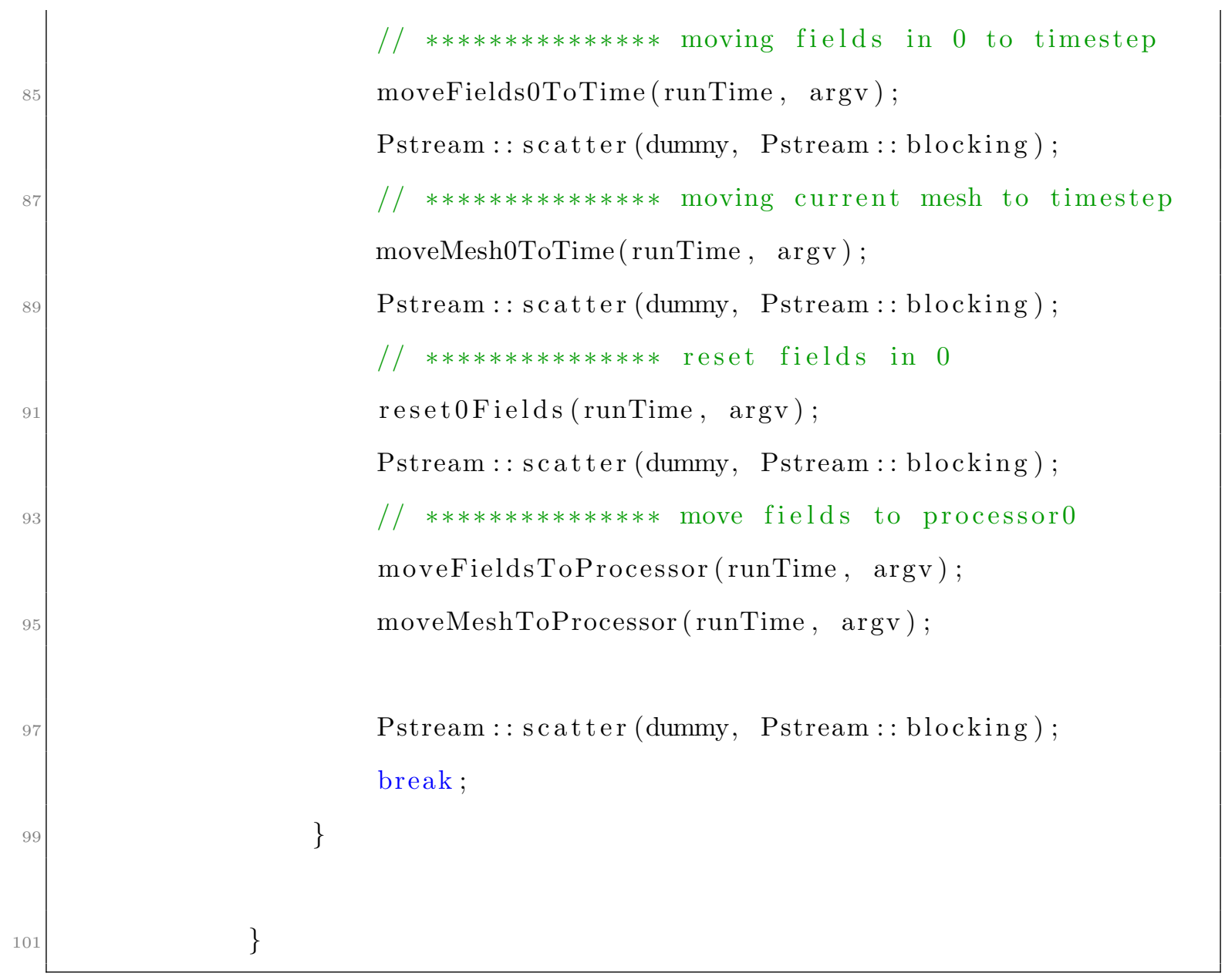

\section{CouplingLoop}




\section{Bibliography}

[1] W.H. Lee. A pressure iteration scheme for two-phase modeling. Technical Report LA-UR 79-975, 1979.

[2] Hyoungsoon Lee, Chirag R. Kharangate, Nikhin Mascarenhas, Ilchung Park, and Issam Mudawar. Experimental and computational investigation of vertical downflow condensation. International Journal of Heat and Mass Transfer, 85:865-879, 2015.

[3] Frdric Gibou, Liguo Chen, Duc Nguyen, and Sanjoy Banerjee. A level set based sharp interface method for the multiphase incompressible navierstokes equations with phase change. Journal of Computational Physics, 222(2):536-555, 2007.

[4] Matthias Mecke, Jochen Winkelmann, and Johann Fischer. Molecular dynamics simulation of the liquidvapor interface: The lennard-jones fluid. The Journal of Chemical Physics, 107(21):9264-9270, 1997.

[5] Jos Alejandre, Dominic J. Tildesley, and Gustavo A. Chapela. Molecular dynamics simulation of the orthobaric densities and surface tension of water. The Journal of Chemical Physics, 102(11):4574-4583, 1995.

[6] M. J. P. Nijmeijer, A. F. Bakker, C. Bruin, and J. H. Sikkenk. A molecular dynamics simulation of the lennard jones liquidvapor interface. The Journal of Chemical Physics, 89(6):3789-3792, 1988. 
[7] Wen-Jing Zhou, Zhi-Qiang Yu, Zhong-Zhen Li, Ya-Ling He, and Wen-Quan Tao. Molecular dynamicscontinuum hybrid simulation for the impingement of droplet on a liquid film. Numerical Heat Transfer, Part A: Applications, 68(5):512-525, 2015.

[8] Ian C. Bourg and Garrison Sposito. Isotopic fractionation of noble gases by diffusion in liquid water: Molecular dynamics simulations and hydrologic applications. Geochimica et Cosmochimica Acta, 72(9):2237-2247, 2008.

[9] V. Kalikmanov. Semiphenomenological theory of the tolman length. Physical Review E, 55(3), 1997.

[10] Thomas Werder, Jens H. Walther, Richard L. Jaffe, Timur Halicioglu, Flavio Noca, and Petros Koumoutsakos. Molecular dynamics simulation of contact angles of water droplets in carbon nanotubes. Nano Letters, 1(12):697-702, 2001.

[11] Takahiro Koishi, Kenji Yasuoka, Shigenori Fujikawa, Toshikazu Ebisuzaki, and Xiao Cheng Zeng. Coexistence and transition between cassie and wenzel state on pillared hydrophobic surface. Proceedings of the National Academy of Sciences, 106(21):8435-8440, 2009.

[12] Takahiro Koishi, Kenji Yasuoka, Shigenori Fujikawa, and Xiao Cheng Zeng. Measurement of contact-angle hysteresis for droplets on nanopillared surface and in the cassie and wenzel states: A molecular dynamics simulation study. ACS Nano, 5(9):6834-6842, 2011.

[13] Robert J. Vrancken, Halim Kusumaatmaja, Ko Hermans, An M. Prenen, Olivier Pierre-Louis, Cees W. M. Bastiaansen, and Dirk J. Broer. Fully reversible transition from wenzel to cassie-baxter states on corrugated superhydrophobic surfaces. Langmuir, 26(5):3335-3341, 2010. 
[14] Toghraie D. Noorian, H. and A. Azimian. Molecular dynamics simulation of poiseuille flow in a rough nano channel with checker surface roughnesses geometry. Heat and Mass Transfer, 50(1), 2013.

[15] S. Sharma and P. Debenedetti. Evaporation rate of water in hydrophobic confinement. Proceedings of the National Academy of Sciences, 109(12), 2012.

[16] J. Hanson B. Chen, J. Molecular dynamics simulations for predicting surface wetting. AIMS Materials Science, 1(2):121, 2014.

[17] Joost H. Weijs, Antonin Marchand, Bruno Andreotti, Detlef Lohse, and Jacco H. Snoeijer. Origin of line tension for a lennard-jones nanodroplet. Physics of Fluids, 23(2):022001, 2011.

[18] V. Carey and Wemhoff. Disjoining pressure effects in ultra-thin liquid films in micropassagescomparison of thermodynamic theory with predictions of molecular dynamics simulations. J. Heat Transfer, 128(12):1276, 2006.

[19] H. Hu and Y. Sun. Molecular dynamics simulations of disjoining pressure effect in ultra-thin water film on a metal surface. Applied Physics Letters, 103(26):263110, 2013.

[20] Chengzhi Hu, Minli Bai, Jizu Lv, and Xiaojie Li. An investigation on the flow and heat transfer characteristics of nanofluids by nonequilibrium molecular dynamics simulations. Numerical Heat Transfer, Part B: Fundamentals, 70(2):152-163, 2016.

[21] S. MARUYAMA and T. KIMURA. A molecular dynamics simulation of bubble nucleation on solid surface. Transactions of the Japan Society of Mechanical Engineers Series B, 65(638):3461-3467, 1999. 
[22] Gyoko Nagayama, Takaharu Tsuruta, and Ping Cheng. Molecular dynamics simulation on bubble formation in a nanochannel. International Journal of Heat and Mass Transfer, 49(2324):4437-4443, 2006.

[23] Shashank Sinha. Molecular dynamics simulation of interfacial tension and contact angle of Lennard-Jones fluid. Thesis, 2004.

[24] John G. Kirkwood and Frank P. Buff. The statistical mechanical theory of surface tension. The Journal of Chemical Physics, 17(3):338-343, 1949.

[25] M. V. Berry, R. F. Durrans, and R. Evans. The calculation of surface tension for simple liquids. J Phys A Lett, 5(1):166-170, 1972.

[26] R.C. Tolman. Journal of Chemical Physics, page 17:333, 1949.

[27] Song Hi Lee. Molecular dynamics simulation of a small drop of liquid argon. Bulletin of the Korean Chemical Society, 33(11):3805-3809, 2012.

[28] A. Amirfazli and A. W. Neumann. Status of the three-phase line tension: a review. Advances in Colloid and Interface Science, 110(3):121-141, 2004.

[29] Gibbs JW. The Scientific Papers of JW Gibbs, volume 1. Dover, New York, 1961.

[30] Rafael Delgado-Buscalioni. Tools for Multiscale Simulation of Liquids Using Open Molecular Dynamics, pages 145-166. Springer Berlin Heidelberg, Berlin, Heidelberg, 2012.

[31] S. O'Connell and P. Thompson. Molecular dynamicscontinuum hybrid computations: A tool for studying complex fluid flows. Physical Review E, 52(6):R5792, 1995. 
[32] Thomas Werder, Jens H. Walther, and Petros Koumoutsakos. Hybrid atomisticcontinuum method for the simulation of dense fluid flows. Journal of Computational Physics, 205(1):373-390, 2005.

[33] T. H. Yen, C. Y. Soong, and P. Y. Tzeng. Hybrid molecular dynamics-continuum simulation for nano/mesoscale channel flows. Microfluidics and Nanofluidics, $3(6): 665-675,2007$.

[34] Jie Sun, Yaling He, Wenquan Tao, Xin Yin, and Huasheng Wang. Roughness effect on flow and thermal boundaries in microchannel/nanochannel flow using molecular dynamics-continuum hybrid simulation. International Journal for $\mathrm{Nu}$ merical Methods in Engineering, 89(1):2-19, 2012.

[35] Kwon O. Hwang, H. and J. Kang. Copper nanocluster diffusion in carbon nanotube. Solid State Communications, 129(11), 2004.

[36] A. Rahman. Correlations in the motion of atoms in liquid argon. Physical Review, 136(2A):A405-A411, 1964.

[37] Steve Plimpton. Fast parallel algorithms for short-range molecular dynamics. Journal of Computational Physics, 117(1):1-19, 1995.

[38] H. Jasak C. Fureby H. G. Weller, G. Tabor. A tensorial approach to computational continuum mechanics using object-oriented techniques. COMPUTERS IN PHYSICS, 12(6), 1998.

[39] He Y. Sun, J. and W. Tao. Molecular dynamicscontinuum hybrid simulation for condensation of gas flow in a microchannel. Microfluidics and Nanofluidics, 7(3), 2009. 
[40] X. B. Nie, S. Y. Chen, W. N. E, and M. O. Robbins. A continuum and molecular dynamics hybrid method for micro- and nano-fluid flow. Journal of Fluid Mechanics, 500:55-64, 2004.

[41] N. Hadjiconstantinou and A. Patera. Heterogeneous atomistic-continuum representations for dense fluid systems. Int. J. Mod. Phys. C, 08(04):967-976, 1997.

[42] N. Hadjiconstantinou and A. Patera. Hybrid atomistic-continuum formulations and the moving contact-line problem. Journal of Computational Physics, 154(2):245-265, 1999.

[43] M. Kalweit and D. Drikakis. Multiscale methods for micro/nano flows and materials. Journal of Computational and Theoretical Nanoscience, 5(9), 2008.

[44] Robert W. Schrage. A theoretical study of interphase mass transfer. Thesis, 1953.

[45] T. Kimura and S. Maruyama. Molecular dynamics simulation of heterogeneous nucleation of a liquid droplet on a solid surface. Microscale Thermophysical Engineering, 6(1):3-13, 2002.

[46] A. Mukherjee and S. Kandlikar. Numerical study of an evaporating meniscus on a moving heated surface. J. Heat Transfer, 128(12):1285, 2006. 